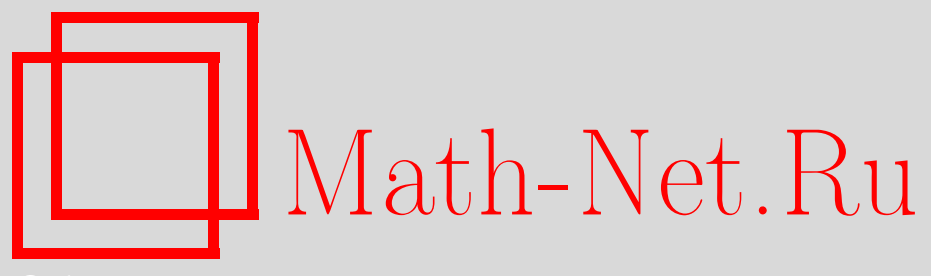

Дж. Харнад, В. З. Энольский, Разложение по функциям Шура $\tau$-функций КП, ассоциированных с алгебраическими кривыми, УМH, 2011, том 66, выпуск 4, 137-178

DOI: https://doi.org/10.4213/rm9435

Использование Общероссийского математического портала Math-Net.Ru подразумевает, что вы прочитали и согласны с пользовательским соглашением http://www . mathnet.ru/rus/agreement

Параметры загрузки:

IP : 3.80 .253 .173

26 апреля 2023 г., 18:09:44

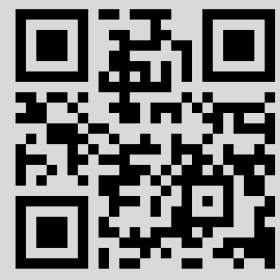




\title{
Разложение по функциям Шура $\tau$-функций КП, ассоциированных с алгебраическими кривыми
}

\author{
Дж. Харнад, В. З. Энольский
}

В работе рассматривается разложение $\tau$-функций КП Сато-СигалаВильсона по функциям Шура. Подробно разобран случай $\tau$-функций, связанных с алгебраическими кривыми произвольного рода. Явные выражения для плюккеровых координат, возникающих как коэффициенты в этом разложении, получены в терминах производных по направлению $\theta$-функции Римана или $\sigma$-функции Клейна вдоль направления потока иерархии КП. С помощью фундаментального бидифференциала показано, что плюккеровы координаты могут быть выражены в виде полиномов от введенных Клейном аналогов для высших родов $\zeta$ - и ю-функций Вейерштрасса. В качестве иллюстрации к развитому в работе подходу детально изложены случаи гиперэллиптических кривых рода два и тригональных кривых рода три.

Библиография: 53 названия.

Ключевые слова: $\tau$-функции, $\sigma$-функции, $\theta$-функции, функции Шура, уравнение Кадомцева-Петвиашвили, алгебро-геометрические решения солитонных уравнений.

\section{СОДЕРЖАНИЕ}

1. Введение.......................................... 138

2. История вопроса. $\tau$-функция Сато-Сигала-Вильсона.............. 144

2.1. Грассманиан гильбертова пространства и плюккеровы коорди-

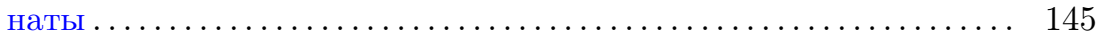

2.2. Абелева группа потока $\Gamma_{+}$и $\tau$-функция КП............... 149

2.3. Разложение в ряд по функциям Шура..................... 150

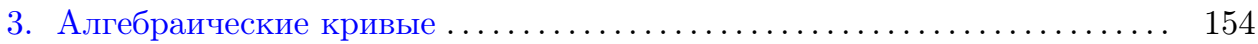

3.1. Функция Бейкера-Ахиезера и $\tau$-функция для алгебраических

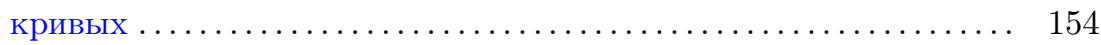

3.2. Последовательности пробелов Вейерштрасса, базисы и фундаментальный бидифференциал . . . . . . . . . . . . . . . . . 159

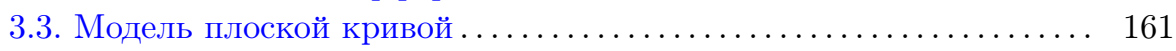

3.4. $\sigma$-функции и алгебро-геометрические формулы для $\pi_{\lambda}(w) \ldots \ldots 163$

Работа частично поддержана Natural Sciences and Engineering Research Council (NSERC), Канада, и фондом FQRNT, Квебек. 
4. Примеры и приложения разложений по функциям Шура . . . . . . . . . . 167

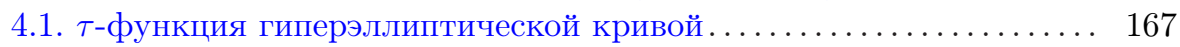

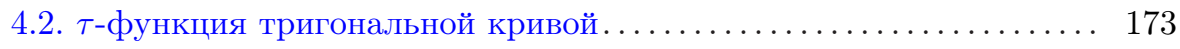

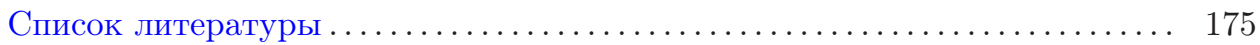

\section{1. Введение}

В середине 1970-х годов С. П. Новиков и Б. А. Дубровин [1]-[3], А. Р. Итс и В. Б. Матвеев [4], [5] (см. также [6]-[9]) применили подход лаксовой пары (изоспектральной деформации) к оператору Хилла для периодических потенциалов с конечнозонным спектром и таким образом определили конечнозонные периодические решения уравнения Кортевега-де Фриза (КдФ)

$$
\mathscr{U}_{t}=6 \mathscr{U} \mathscr{U}_{x}-\mathscr{U}_{x x x} .
$$

Итс и Матвеев открыли замечательную формулу, явно задающую периодические и квазипериодические решения уравнения КдФ как вторую логарифмическую производную $\theta$-функции Римана:

$$
\mathscr{U}(x, t)=-\frac{\partial^{2}}{\partial x^{2}} \ln \theta(\mathbf{U} x+\mathbf{V} t+\mathbf{W})+C,
$$

где $\mathbf{U}, \mathbf{V}, \mathbf{W}=$ const $\in \mathbb{C}^{g}$ и $C \in \mathbb{C}$. Здесь $\theta$-функция определена решеткой периодов гиперэллиптической кривой $X$ произвольного рода $g$, а "обмоточные векторы" $\mathbf{U}, \mathbf{V}$ - периоды абелевых дифференциалов второго рода. Этот результат следует из более общей формулы (3.7) для ассоциированной функции Бейкера-Ахиезера (см. (2.61)), которая применима для иерархии КП и впервые получена Итсом в случае КдФ (она появляется в приложении к [10]).

Вышеизложенное составило важную часть общей теории алгебро-геометрических решений уравнения КдФ (см., например, обзор [10]). И. М. Кричевер [11] распространил эти соображения в более общем случае на квазипериодические решения иерархии КП, что привело к общему методу решения таких уравнений в частных производных, определяемых заданием алгебраической кривой и некоторой дополнительной информации о ней. (Обзор этого подхода и дальнейшие приложения см. в [12].) Эти результаты оказали большое влияние на последующие разработки в теории интегрируемых нелинейных иерархий и их приложения в различных областях математики и физики.

Феномен алгебро-геометрической интегрируемости был рассмотрен с разных точек зрения. В этой работе мы обсуждаем теорию $\tau$-функций такой, какой она сформулирована у М. и Я. Сато [13]-[15] и была развита в работах М. Сато, Э. Дейта, М. Джимбо, М. Касивара, Т. Мива и др. (см., например, [16]). Мы также используем геометрическую формулировку Г. Сигала и Дж. Вильсона [17]. В этом подходе $\tau$-функция

$$
\tau=\tau_{w}(\mathbf{t}), \quad \tau_{w}(\mathbf{0}) \neq 0,
$$

считается зависящей от двух наборов переменных - бесконечномерного вектоpa $\mathbf{t}=\left(t_{1}, t_{2}, \ldots\right) \in \mathbb{C}^{\infty}$ и элемента $w \in \operatorname{Gr}_{\mathscr{H}_{+}}(\mathscr{H})$ бесконечномерного грассманиана, состоящего из подпространств поляризованного гильбертова про- 
странства (прямой суммы двух взаимно ортогональных линейно изоморфных подпространств)

$$
\mathscr{H}=\mathscr{H}_{+}+\mathscr{H}_{-},
$$

“соизмеримых" с фиксированным подпространством $\mathscr{H}_{+}$(т. е. ортогональная проекция на $\mathscr{H}_{+}$является "большой" - фредгольмовым оператором, в то время как проекция на $\mathscr{H}_{-}$, например, лежит в классе Гильберта-Шмидта). Плюккеровы соотношения, определяющие вложение $\operatorname{Gr}_{\mathscr{H}}(\mathscr{H})$ как подмногообразия проективизации бесконечного внешнего пространства $\mathbf{P}(\Lambda \mathscr{H})$, эквивалентны бесконечному набору билинейных дифференциальных соотношений на переменные $\mathbf{t}$, а именно уравнениям Хироты, из которых, в свою очередь, следуют уравнения иерархии КП.

В частном случае, который мы рассматриваем в этой работе, $w$ определяется некоторыми алгебро-геометрическими данными, а именно данными Дубровина-Кричевера-Новикова (далее ДКН), состоящими из алгебраической кривой $X$ рода $g$, эффективного неспециального дивизора степени $g$ :

$$
\mathscr{D}=\sum_{i=1}^{g} p_{i}, \quad p_{i} \in X,
$$

точки $p_{\infty}$ на "бесконечности" и локального параметра $\xi=1 / z$ с $\xi\left(p_{\infty}\right)=0$.

Затем мы выбираем базис гомологий $\left\{\mathfrak{a}_{1}, \ldots, \mathfrak{a}_{g} ; \mathfrak{b}_{1}, \ldots, \mathfrak{b}_{g}\right\}$ для $X$, состоящий из $\mathfrak{a}$ - и $\mathfrak{b}$-циклов, удовлетворяющих условиям пересечения

$$
\mathfrak{a}_{i} \circ \mathfrak{a}_{j}=0, \quad \mathfrak{b}_{i} \circ \mathfrak{b}_{j}=0, \quad \mathfrak{a}_{i} \circ \mathfrak{b}_{j}=\delta_{i j},
$$

а также нормированный базис $\left\{u_{1}, \ldots, u_{g}\right\}$ голоморфных абелевых дифференциалов и каноническое разрезание (полигонизацию) кривой $X$, полученное разрезанием вдоль $\mathfrak{a}$ - и $\mathfrak{b}$-циклов. После выбора произвольной базисной точки $p_{0}$ отображение Абеля

$$
\mathscr{A}: \mathscr{S}^{g} X \rightarrow \mathscr{J}(X)=\mathbb{C}^{g} / \Gamma
$$

из $g$-й симметрической степени кривой $X$ в ее многообразие Якоби (фактор $\mathbb{C}^{g}$ по решетке периодов) определяется интегрированием этих дифференциалов от базисной точки до нужных точек. Данные ДКН определяют вектор $\mathbf{e}=$ $\left(e_{1}, \ldots, e_{g}\right)^{T} \in \mathbb{C}^{g}$ как образ дивизора $\mathscr{D}$ при отображении Абеля (с точностью до полигонизации)

$$
\mathbf{e}=\mathscr{A}(\mathscr{D})-\mathscr{A}\left(p_{\infty}\right)+\mathbf{K},
$$

сдвинутом на константу Римана $\mathbf{K}$, соответствующую полигонизации. Мы можем теперь кратко обозначить соответствующую $\tau$-функцию как $\tau(\mathbf{e}, \mathbf{t})$.

$\tau$-функция может быть представлена в виде ряда Тейлора от $\mathbf{t}$ и затем разложена по базису, состоящему из функций Шура $s_{\lambda}(\mathbf{t})$, параметризованных разбиениями $\lambda=\left(\lambda_{1}, \lambda_{2}, \ldots, \lambda_{\ell(\lambda)}\right)$ (где $\lambda_{i}$ образуют нестрого убывающую последовательность неотрицательных целых чисел $\lambda_{1} \geqslant \lambda_{2} \geqslant \cdots$ с ненулевым последним членом $\lambda_{\ell(\lambda)}$, где $\ell(\lambda)-$ длина $\left.\lambda\right)$. Параметры тока $\left(t_{1}, t_{2}, \ldots\right)$ могут быть отождествлены с мономиальными суммами

$$
t_{i}=\frac{1}{i} \sum_{a=1}^{N} x_{a}^{i}
$$


$N$ вспомогательных переменных для любого $N \geqslant \ell(\lambda)$ посредством перехода к (устойчивому) пределу $N \rightarrow \infty$.

Тождество Коши-Литтлвуда [18] (эквивалентное свойству абелевой группы потока КП) позволяет нам записать это разложение по базису в виде

$$
\tau(\mathbf{e}, \mathbf{t})=\left.\sum_{\lambda}\left[s_{\lambda}\left(\frac{\partial}{\partial t_{1}}, \ldots, \frac{1}{k} \frac{\partial}{\partial t_{k}}, \ldots\right) \tau(\mathbf{e}, \mathbf{t})\right]\right|_{\mathbf{t}=\mathbf{0}} s_{\lambda}(\mathbf{t}),
$$

где суммирование идет по всем разбиениям $\lambda$. Важно отметить, что коэффициенты в этом разложении,

$$
\pi_{\lambda}(w):=\left.\left[s_{\lambda}\left(\frac{\partial}{\partial t_{1}}, \ldots, \frac{1}{k} \frac{\partial}{\partial t_{k}}, \ldots\right) \tau(\mathbf{e}, \mathbf{t})\right]\right|_{\mathbf{t}=\mathbf{0}},
$$

являются в точности плюккеровыми координатами элемента $w \in \operatorname{Gr}_{\mathscr{H}_{+}}(\mathscr{H})$ при плюккеровом вложении

$$
\mathfrak{P}: \operatorname{Gr}_{\mathscr{H}}(\mathscr{H}) \rightarrow \mathbf{P}(\mathscr{F})
$$

в проективизацию внешнего пространства

$$
\mathscr{F}=\Lambda \mathscr{H},
$$

которое является пополнением пространства сумм, над базисом, состоящим из полубесконечных внешних произведений базисных элементов из $\mathscr{H}$ (фермионного фоковского пространства). В этой постановке билинейные соотношения Хироты иерархии КП эквивалентны плюккеровым соотношениям, которым удовлетворяют коэффициенты $\left\{\pi_{\lambda}(w)\right\}$.

Каждому разбиению $\lambda=\left(\lambda_{1}, \lambda_{2}, \ldots\right)$ мы ставим в соответствие, как обычно, диаграмму Юнга с $\lambda_{1}$ клетками в первом ряду, $\lambda_{2}$ клетками во втором и т. д. Например,

$$
\lambda=(3,3,1) \Leftrightarrow \begin{array}{|l|l|l|}
\hline & & \\
\hline & & \\
\hline & &
\end{array}
$$

Разбиения вида $(k, \underbrace{1,1, \ldots, 1}_{j}) \equiv\left(k, 1^{j}\right)$ называются крюками. Разбиения могут быть эквивалентным образом представлены в обозначениях Фробениуса как

$$
\lambda \sim\left(a_{1}, \ldots, a_{k} \mid b_{1}, \ldots, b_{k}\right),
$$

где $k$ - число диагональных ячеек в диаграмме Юнга, называемое рангом разбиения, а числа $a_{i}$ и числа $b_{i}$ - количество ячеек соответственно справа и снизу от диагональных ячеек [19]. Формула Джамбелли

$$
s_{\left(a_{1}, \ldots, a_{k} \mid b_{1}, \ldots, b_{k}\right)}=\operatorname{det}\left(s_{\left(a_{i} \mid b_{j}\right)}\right)
$$

выражает функцию Шура, соответствующую произвольному разбиению, как детерминант матрицы, элементы которой суть функции Шура, соответствующие только диаграммам-крюкам. М. Сато также использовал формулу Джамбелли в разложении коэффициентов выражения (1.10), так как верны те же 
самые детерминантные соотношения, записанные для плюккеровых координат образа любого элемента $w$ грассманиана (т. е. для любого полностью разложимого элемента из $\Lambda \mathscr{H}$ ) в терминах координат разбиений-крюков (см. (2.23)). Это составляет явное решение плюккеровых соотношений, применимое на аффинной окрестности, соответствующей “большой клетке". Согласно (1.11), peзультирующие соотношения возникают как уравнения в частных производных, для которых $\tau$-функция играет роль производящей функции.

Такие разложения в ряд справедливы для любой $\tau$-функции $\tau_{w}(\mathbf{t})$, но здесь мы в основном будем рассматривать $\tau$-функции $\tau(\mathbf{e}, \mathbf{t})$, ассоциированные с данными ДКН на алгебраической кривой $X$ рода $g$, и вычислим коэффициенты разложения по функциям Шура в этом случае.

Пусть кривая $X$ снабжена каноническим базисом гомологий $\left(\mathfrak{a}_{1}, \ldots, \mathfrak{a}_{g}\right.$; $\left.\mathfrak{b}_{1}, \ldots, \mathfrak{b}_{g}\right)$ и соответствующей полигонизацией, выберем базис голоморфных дифференциалов $\mathbf{u}=\left(u_{1}, \ldots, u_{g}\right)^{T}$, упорядоченных согласно степени их обращения в нуль в точке Вейерштрасса $p_{\infty}$ в бесконечности, $n_{g}+1, \ldots, n_{1}+1$, где $\mathfrak{W}=\left(n_{1}, \ldots, n_{g}\right)-$ последовательность пробелов Вейерштрасса в $p_{\infty}$ (см. п. 3.2).

Обозначим через $\mathfrak{A}$ и $\mathfrak{B}$ матрицы периодов:

$$
\mathfrak{A}=\left(\oint_{\mathfrak{a}_{\mathfrak{j}}} u_{i}\right)_{i, j=1, \ldots, g}, \quad \mathfrak{B}=\left(\oint_{\mathfrak{b}_{\mathfrak{j}}} u_{i}\right)_{i, j=1, \ldots, g} .
$$

Тогда якобиан кривой $\mathrm{Jac}(X)$ определяется как $\mathbb{C}^{g} / \mathfrak{A} \oplus \mathfrak{B}$.

Будем говорить о $(\mathfrak{A}, \mathfrak{B})$ как о первых матрицах периодов или римановых матрицах периодов. Вторые матрицы периодов, (, T), образованы подобным образом из периодов мероморфных дифференциалов $\mathbf{r}=\left(r_{1}, \ldots, r_{g}\right)^{T}$ с полюсами только в точках Вейерштрасса $p_{\infty}$, порядков $n_{g}+1, \ldots, n_{1}+1$ соответственно. Матрицы

$$
\mathfrak{S}=-\left(\oint_{\mathfrak{a}_{j}} r_{i}\right)_{i, j=1, \ldots, g}, \quad \mathfrak{T}=-\left(\oint_{\mathfrak{b}_{j}} r_{i}\right)_{i, j=1, \ldots, g}
$$

нормированы условием (обобщенным уравнением Лежандра)

$$
\left(\begin{array}{cc}
\mathfrak{A} & \mathfrak{B} \\
\mathfrak{S} & \mathfrak{T}
\end{array}\right) \mathbf{J}\left(\begin{array}{cc}
\mathfrak{A} & \mathfrak{B} \\
\mathfrak{S} & \mathfrak{T}
\end{array}\right)^{T}=-2 \imath \pi \mathbf{J}, \quad \mathbf{J}=\left(\begin{array}{cc}
\mathbf{0}_{g} & -\mathbf{1}_{g} \\
\mathbf{1}_{g} & \mathbf{0}_{g}
\end{array}\right) .
$$

Матрица

$$
\varkappa:=\mathfrak{S A}^{-1}
$$

с необходимостью симметрична, $\varkappa^{T}=\varkappa$, и

$$
\mathfrak{S}=\varkappa \mathfrak{A}, \quad \mathfrak{T}=\varkappa \mathfrak{B}-\frac{\imath \pi}{2}\left(\mathfrak{A}^{-1}\right)^{T} .
$$

ЗАмЕчАниЕ 1.1. В базисе $(\mathbf{u}, \mathbf{r})$ мероморфные дифференциалы $\mathbf{r}$ определены не однозначно, а только с точностью до прибавления голоморфного дифференциала. Поэтому матрица $\varkappa$ определена только с точностью до прибавления произвольной симметричной матрицы. Тем не менее, для наших целей достаточно выбрать конкретного представителя класса дифференциалов $\mathbf{r}$, и таковой будет указан в каждом случае, рассмотренном детально в примерах. 
Следуя Г. Ф. Бейкеру [20], мы ассоциируем с кривой $X$ фундаментальный бидифференциал $\Omega(p, q)$, который является единственной симметричной мероморфной 2-формой на $X \times X$ с полюсом второго порядка на диагонали $p=q$, а вне диагонали голоморфной по каждой переменной. Локально он принимает вид

$$
\Omega(p, q)=\frac{\mathrm{d} \xi(p) \mathrm{d} \xi(q)}{(\xi(p)-\xi(q))^{2}}+\sum_{i, j=0}^{\infty} \mu_{i j}\left(p_{0}\right) \xi(p)^{i} \xi(q)^{j} \mathrm{~d} \xi(p) \mathrm{d} \xi(q),
$$

где $\xi(p)$ и $\xi(q)$ - локальные координаты в окрестности базисной точки $p_{0}$, $\xi\left(p_{0}\right)=0$, а коэффициенты $\mu_{i j}\left(p_{0}\right)$ являются симметричными по индексам $i$ и $j: \mu_{i j}\left(p_{0}\right)=\mu_{j i}\left(p_{0}\right)$. 2-форма $\Omega(p, q)$ нормирована соотношениями

$$
\oint_{\mathfrak{a}_{j}} \Omega(p, q)=0, \quad j=1, \ldots, g .
$$

Обычно $\Omega(p, q)$ реализуется как вторая логарифмическая производная примформы или $\theta$-функции [21], [22]. Но в нашем подходе мы используем альтернативное представление $\Omega(p, q)$ в алгебрачческой форме, которая восходит к Вейерштрассу и Клейну, как описано Бейкером в [23]:

$$
\Omega(p, q)=\frac{\mathscr{F}(p, q)}{P_{y}(p) P_{w}(q)(x-z)^{2}} \mathrm{~d} x \mathrm{~d} z+2 \mathbf{u}(p)^{T} \varkappa \mathbf{u}(q),
$$

где $p=(x, y), q=(z, w)$, функция $\mathscr{F}(p, q)=\mathscr{F}((x, y),(z, w))$ является полиномом от своих аргументов с коэффициентами, зависящими от параметров, явно определяющих кривую $X$ как плоскую модель, заданную полиномиальным уравнением

$$
P(x, y)=0,
$$

u является $g$-компонентным вектором, состоящим из голоморфных дифференциалов, а $\varkappa$ - симметричная матрица (1.20), задающая нормировку (1.23) бидифференциала $\Omega(p, q)$. Мы будем говорить о первом члене в правой части (1.24), который включает полином $\mathscr{F}(p, q)$, как об алгебраической части ${ }^{1}$ и обозначать ее $\Omega^{\mathrm{alg}}(p, q){ }^{2} \mathrm{~B}$ окрестности базисной точки $p_{0}, \xi\left(p_{0}\right)=0$, форма $\Omega^{\mathrm{alg}}(p, q)$ разлагается в степенной ряд:

$$
\Omega^{\mathrm{alg}}(p, q)=\frac{\mathrm{d} \xi(p) \mathrm{d} \xi(q)}{(\xi(p)-\xi(q))^{2}}+\sum_{i, j=0}^{\infty} \mu_{i j}^{\mathrm{alg}}\left(p_{0}\right) \xi(p)^{i} \xi(q)^{j} \mathrm{~d} \xi(p) \mathrm{d} \xi(q),
$$

где величины $\mu_{i j}^{\text {alg }}\left(p_{0}\right)$ являются алгебраическими функциями от $\xi\left(p_{0}\right)$ и коэффициентов кривой. Трансцендентная часть $\Omega^{\text {trans }}(p, q)$ бидифференциала $\Omega(p, q)$ голоморфна, и ее разложение в ряд в окрестности базисной точки $p_{0}$ имеет вид

$$
\Omega^{\mathrm{trans}}(p, q) \equiv 2 \mathbf{u}(p)^{T} \varkappa \mathbf{u}(q)=\sum_{i, j=0}^{\infty} \mu_{i j}^{\mathrm{trans}}\left(p_{0}\right) \xi(p)^{i} \xi(q)^{j} \mathrm{~d} \xi(p) \mathrm{d} \xi(q) .
$$

\footnotetext{
${ }^{1}$ Множитель 2 в нормировке делает случай $g=1$ согласованным с обычным случаем в теории эллиптических функций Вейерштрасса.

${ }^{2}$ Представление (1.24) развито далее В. М. Бухштабером, Д. В. Лейкиным и одним из авторов [24] и в последнее время А. Накаяшики [25].
} 
Поэтому

$$
\Omega(p, q)=\Omega^{\mathrm{alg}}(p, q)+\Omega^{\mathrm{trans}}(p, q)
$$

и

$$
\mu_{i j}\left(p_{0}\right)=\mu_{i j}^{\mathrm{alg}}\left(p_{0}\right)+\mu_{i j}^{\mathrm{trans}}\left(p_{0}\right)
$$

для всех $p_{0}$ и $i, j \in \mathbb{Z}$.

На описанном выше алгебраическом представлении фундаментального дифференциала основано определение многомерной $\sigma$-функции в терминах многомерной (римановой) $\theta$-функции $\theta$. Она отличается от $\theta$ экспоненциальным множителем и модулярным множителем $C$ :

$$
\sigma(\mathbf{v})=C \exp \left\{\frac{1}{2} \mathbf{v}^{T} \varkappa \mathbf{v}\right\} \theta\left(\mathfrak{A}^{-1} \mathbf{v} ; \tau\right)
$$

где $\varkappa$ и $\mathfrak{A}$ определены в (1.20) и (1.17). Эти изменения делают $\sigma(\mathbf{v})$ инвариантной относительно действия симплектической группы, таким образом, для любого $\gamma \in \operatorname{Sp}(2 g, \mathbb{Z})$ имеем:

$$
\sigma(\mathbf{v} ; \gamma \circ \mathfrak{M})=\sigma(\mathbf{v} ; \mathfrak{M})
$$

где $\mathfrak{M}$ - набор периодов кривой $X$. Многомерная $\sigma$-функция является естественным обобщением $\sigma$-функции Вейерштрасса на алгебраические кривые высших родов.

ЗАмЕчАниЕ 1.2. В своих лекциях [26] Вейерштрасс определил $\sigma$-функцию как ряд, коэффициенты которого заданы рекуррентно, что явилось ключевым моментом теории эллиптических функций Вейерштрасса. Обобщение этого результата на кривые рода два было начато Бейкером [20] и недавно завершено Бухштабером и Лейкиным [27], получившими рекуррентные соотношения на коэффициенты ряда для $\sigma$-функции в виде замкнутой системы. Также Бухштабер и Лейкин недавно нашли операторную алгебру, аннулирующую $\sigma$-функцию $(n, s)$-кривых высших родов [28]. Нахождение соответствующего рекуррентного определения $\sigma$-функций высших родов в стиле работы [28] остается перспективной задачей.

В данной работе мы изучаем связи между многомерной $\sigma$-функцией и $\tau$-функцией Сато [13]--[15] для случая квазипериодических решений, ассоциированных с данными ДКН на алгебраической кривой. Таким образом, в основном мы рассматриваем этот класс "алгебро-геометрических $\tau$-функциональных" решений. Они по существу те же, что и решения исследованные Феем [29], [30] в терминах $\theta$-функций. Такие $\tau$-функции могут быть выражены через $\sigma$-функции следующим образом (см. предложение 3.1$)^{3}$ :

$$
\frac{\tau(\mathbf{e}, \mathbf{t})}{\tau(\mathbf{e}, \mathbf{0})}=\frac{\sigma\left(\sum_{k=1}^{\infty} \mathfrak{A} \mathbf{U}_{k}\left(p_{\infty}\right) t_{k}+\mathfrak{A} \mathbf{e}\right)}{\sigma(\mathfrak{A} \mathbf{e})} \exp \left\{\frac{1}{2} \sum_{k, l=0}^{\infty} \mu_{k l}^{\operatorname{alg}}\left(p_{\infty}\right) t_{k} t_{l}\right\} .
$$

Здесь, как и выше, $\mathfrak{A}$ - матрица периодов голоморфных дифференциалов, $\mathbf{U}_{k}\left(p_{\infty}\right), k=1,2, \ldots,-$ "обмоточные векторы", т. е. $\mathfrak{b}$-периоды нормированных

\footnotetext{
${ }^{3}$ Недавно Накаяшики [31] независимо предложил подобное выражение для алгебро-геометрических $\tau$-функций в терминах многомерной $\sigma$-функции и изучил свойства $\sigma$-ряда.
} 
дифференциалов второго рода с полюсами порядка $k+1$ в точке $p_{\infty}$, a $\mathbf{e}-$ произвольная точка многообразия Якоби $\operatorname{Jac}(X)$.

ЗАмечание 1.3. В серии работ [32]-[34] В. М. Бухштабер и С. Ю. Шорина также изучили связи между $\theta$-функциональными и $\sigma$-функциональными решениями иерархии КдФ.

Работа упорядочена следующим образом. В разделе 2 мы рассматриваем геометрическую формулировку $\tau$-функции Сато-Сигала-Вильсона в терминах грассманианов гильбертова пространства. Выводится интерпретация коэффициентов разложения по функциям Шура как плюккеровых координат (предложение 2.2), а также их выражение в терминах аффинных координат на большой клетке и разбиений-крюков (следствие 2.3).

В разделе 3 мы ограничиваемся специальным случаем $\tau$-функций, ассоциированных с данными ДКН на алгебраической кривой. Явная формула для таких $\tau$-функций в терминах $\theta$-функций Римана дана в (3.37). Аффинные координаты, задающие разложение по функциям Шура, явно вычисляются (см. (3.31)) в терминах производных по направлению $\theta$-функции Римана вдоль направлений потока. В п. 3.2 мы рассматриваем теорему Вейерштрасса о пробелах и вводим нормированный симметричный бидифференциал $\Omega(p, q)$. Это позволяет нам сделать явным разделение на "алгебраическую" и "трансцендентную" части (следствие 3.1) бесконечной квадратичной формы $Q$, возникающей в экспоненциальном члене в (3.37) и в формуле $(3.31)$, определяющей аффинные координаты. Эквивалентное выражение для $\tau$-функции в терминах $\sigma$-функции, явно выражающее ее свойства при модулярном преобразовании, выводится в предложении 3.1, формула (3.77). Отсюда выводится метод явного вычисления матричных элементов матрицы аффинных координат как полиномов от функций Клейна $\left\{\zeta_{i}, \wp_{i j}\right\}$, которые обобщают $\zeta$ - и ю-функции Вейерштрасса на кривые произвольного рода.

В разделе 4 мы рассматриваем несколько примеров, для которых аффинные координаты вычисляются явно, и используем плюккеровы соотношения для вывода тождеств, связывающих $\zeta$ - и ю-функции Клейна различных степеней. Случай гиперэллиптических кривых рассмотрен детально, явные формулы получены в случае рода $g=2$. В предложении 4.1 мы выводим алгебраические соотношения между ю-функциями Клейна разных порядков для этого случая. Рассмотрен следующий пример, основанный на модели некоторого класса плоских тригональных кривых, и даны явные выражения для простейших матричных элементов матрицы аффинных координат, вычисленных в терминах $\zeta$ - и ю-функций Клейна для этого случая, а также тождества, связывающие ю-функции разных порядков, которые снова следуют из плюккеровых соотношений.

\section{2. История вопроса. $\tau$-функция Сато-Сигала-Вильсона}

Последующее является кратким изложением подходов Сато [15], а также Сигала и Вильсона [17] к $\tau$-функциям КП. Первый является существенно алгебраическим по сути, второй - функционально-аналитическим, но мы комбинируем 
элементы обоих подходов. За более точными определениями основных составляющих частей (грассманианы гильбертова пространства, бесконечные группы преобразований, линейные расслоения детерминантов, фермионное фоковское пространство, плюккерово отображение и т. д.) читатель может обратиться к этим двум первоисточникам, которые согласованы в геометрической структуpe, но не в аналитических деталях. Вводное изложение, данное ниже, мы старались сделать простым и самодостаточным - насколько это возможно, а также применимым к рассматриваемым случаям. Функционально-аналитические детали общей постановки задачи либо опускаются, либо опираются на [17], а составляющие части, по возможности, сделаны похожими на конечномерный случай.

\section{1. Грассманиан гильбертова пространства и плюккеровы коор-} динаты. Следуя [17], мы начинаем с гильбертова пространства

$$
\mathscr{H}:=L^{2}\left(S^{1}\right)=\mathscr{H}_{+} \oplus \mathscr{H}_{-}
$$

комплекснозначных функций $f$ на единичной окружности $\left\{z=e^{i \phi}\right\}$ в комплексной плоскости, квадраты которых интегрируемы и которые могут быть разложены в сумму

$$
f=f_{+}+f_{-}, \quad f_{ \pm} \in \mathscr{H}_{ \pm},
$$

где $f_{ \pm} \in \mathscr{H}_{ \pm}-$положительная и отрицательная части ряда Фурье.

Иными словами, $\mathscr{H}_{+}$можно интерпретировать как пространство голоморфных функций на внутренности единичного круга и $\mathscr{H}_{-}-$как пространство голоморфных функций вне круга, обращающихся в нуль при $z=\infty$, с ортонормированными базисами из мономов по $z$ :

$$
\mathscr{H}_{+}=\operatorname{span}\left\{e_{j}:=z^{-j-1}\right\}_{j=-1,-2, \ldots}, \quad \mathscr{H}_{-}=\operatorname{span}\left\{e_{j}:=z^{-j-1}\right\}_{j=0,1,2, \ldots} .
$$

ЗАмечАние 2.1. Соглашение обозначать базисные векторы $e_{j}$ таким образом, что $\mathscr{H}_{+}$является линейной оболочкой векторов с отрицательными индексами $j$, выбрано так, чтобы при плюккеровом отображении (см. ниже) $\mathscr{H}_{+}$переводилось в $|0\rangle=e_{-1} \wedge e_{-2} \wedge \cdots$, являющееся "дираковским морем", в котором все состояния с отрицательной "энергией” заняты.

Мы обозначаем через $\operatorname{Gr}_{\mathscr{H}_{+}}(\mathscr{H})$ грассманиан гильбертова пространства, точки которого - замкнутые подпространства $w \subset \mathscr{H}$, соизмеримые с $\mathscr{H}_{+}$ в том смысле, что ортогональная проекция $\pi^{\perp}: w \rightarrow \mathscr{H}_{+}$на $\mathscr{H}_{+}$вдоль $\mathscr{H}_{-}$ является фредгольмовым отображением индекса нуль и ортогональная проекция $\pi^{\perp}: w \rightarrow \mathscr{H}_{-}$на $\mathscr{H}_{-}$вдоль $\mathscr{H}_{+}$принадлежит классу Гильберта-Шмидта. (В [17] он называется компонентой виртуалъной размерности нуль полного грассманиана гильбертова пространства.)

Пусть

$$
\begin{aligned}
w & =\operatorname{span}\left\{w_{j}\right\}_{j \in \mathbb{N}}, \\
w_{j} & =\sum_{i \in \mathbb{Z}} w_{i j} e_{i} .
\end{aligned}
$$


Относительно мономиального базиса $\left\{e_{j}\right\}$ репер $\left\{w_{0}, w_{1}, \ldots\right\}$ может быть представлен как бесконечная матрица $W$ с компонентами $\left\{w_{i j}\right\}_{i \in \mathbb{Z}, j \in \mathbb{N}}, j$-й столбец $W_{j}$ которой имеет компоненты $\left\{w_{i j}\right\}_{i \in \mathbb{Z}}$ :

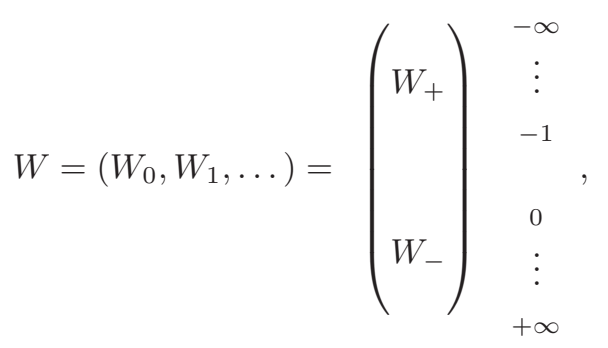

где строки занумерованы целыми числами, возрастающими при движении вниз, с нулевой строкой наверху блока $W_{-}$, а столбцы занумерованы неотрицательными целыми числами, начинающимися с нуля слева и возрастающими при движении направо.

ЗАмечАниЕ 2.2. Отметим, что способ нумерации столбцов соответствует мономиальным степеням в $\mathscr{H}_{+}$, тогда как способ нумерации строк соответствует базисным элементам $\left\{e_{j}\right\}$.

Здесь $W_{-}$и $W_{+}$могут рассматриваться как представители соответственно отображений $w_{-}: \mathscr{H}_{+} \rightarrow \mathscr{H}_{-}$и $w_{+}: \mathscr{H}_{+} \rightarrow \mathscr{H}_{+}$таких, что $w-$ образ их суммы:

$$
w=\left(w_{-}+w_{+}\right)\left(\mathscr{H}_{+}\right) .
$$

Грассманиан $\operatorname{Gr}_{\mathscr{H}_{+}}(\mathscr{H})$ можно интерпретировать как фактор расслоения $\operatorname{Fr}_{\mathscr{H}_{+}}(\mathscr{H})$ допустимых реперов по правому действию группы $\mathfrak{G l}\left(\mathscr{H}_{+}\right)$линейных преобразований базиса. (См. в [17] точное определение, согласно которому у элементов $\mathfrak{G l}\left(\mathscr{H}_{+}\right)$должен быть ненулевой конечный детерминант.) Таким образом, $\operatorname{Fr}_{\mathscr{H}_{+}}(\mathscr{H})$ можно рассматривать, подобно конечномерному случаю, как главное $\mathfrak{G l}\left(\mathscr{H}_{+}\right)$-расслоение над $\operatorname{Gr}_{\mathscr{H}_{+}}(\mathscr{H})$, с которым мы можем ассоциировать посредством детерминантного представления линейное расслоение Det $\rightarrow$ $\operatorname{Gr}_{\mathscr{H}_{+}}(\mathscr{H})$ и двойственное к нему $\operatorname{Det}^{*} \rightarrow \operatorname{Gr}_{\mathscr{H}_{+}}(\mathscr{H})$. Голоморфное сечение последнего определяется разбиением $\lambda=\left(\lambda_{1} \geqslant \lambda_{2} \geqslant \cdots \geqslant \lambda_{\ell(\lambda)}>0\right), \lambda_{i} \in \mathbb{N}$, где $\ell(\lambda)$ обозначает длину, подобно конечномерному случаю. Расширим множество частей $\left\{\lambda_{j}\right\}_{j=1, \ldots, \ell(\lambda)}$ до бесконечной последовательности $\left\{\lambda_{j}\right\}_{j=1, \ldots, \infty}$ обычным способом [18], полагая

$$
\lambda_{j}=0, \text { если } j>\ell(\lambda) .
$$

Детерминант $\operatorname{det}\left(W_{\lambda}\right)$ подматрицы $W_{\lambda}$ матрицы $W$, состоящей из строк $\left\{\lambda_{i}-i\right\}_{i \in \mathbb{N}}$, определяет голоморфные сечения $\sigma: \operatorname{Gr}_{\mathscr{H}_{+}}(\mathscr{H}) \rightarrow$ Det* $^{*}$ расслоения Det $^{*} \rightarrow \operatorname{Gr}_{\mathscr{H}_{+}}(\mathscr{H})$, которые порождают пространство допустимых сечений. (За аналитическими подробностями, необходимыми для определения класса допустимых сечений, мы снова отсылаем читателя к [17].)

Как и в конечномерном случае, пространство таких голоморфных сечений может быть отождествлено с некоторым подпространством $\mathscr{F}_{0} \subset \mathscr{F}$ внешнего пространства $\mathscr{F}:=\Lambda \mathscr{H}$, "сектором нулевого заряда" пространства $\mathscr{F}$, которое 
можно интерпретировать как полное "фермионное фоковское пространство". Пусть $\mathscr{F}_{0}$ - линейная оболочка внешних элементов

$$
|\lambda\rangle:=e_{l_{1}} \wedge e_{l_{2}} \wedge e_{l_{3}} \wedge \cdots,
$$

где $l_{i}$-е для каждого разбиения $\lambda=\left(\lambda_{1}, \lambda_{2}, \ldots\right)$ - это “координаты частиц":

$$
l_{i}=\lambda_{i}-i
$$

Внешние элементы образуют ортонормированный базис относительно внутреннего произведения, индуцированного на $\mathscr{F}_{0} \subset \Lambda \mathscr{H}$ произведением на $\mathscr{H}$.

Для каждого разбиения $\lambda$ мы можем также определить $\mathscr{H}_{\lambda} \in \operatorname{Gr}_{\mathscr{H}_{+}}(\mathscr{H})$ формулой

$$
\mathscr{H}_{\lambda}=\operatorname{span}\left\{e_{l_{i}}\right\}_{i \in \mathbb{N}} .
$$

В частности, элемент $\mathscr{H}_{0}$, соответствующий тривиальному разбиению $\lambda=0$, есть $\mathscr{H}_{+}$. Определим плюккерово отображение $\widehat{\mathfrak{P}}: \operatorname{Fr}_{\mathscr{H}_{+}}(\mathscr{H}) \rightarrow \mathscr{F}_{0}$ естественным образом:

$$
\begin{aligned}
& \widehat{\mathfrak{P}}: \operatorname{Fr}_{\mathscr{H}_{+}}(\mathscr{H}) \rightarrow \mathscr{F}_{0}, \\
& \widehat{\mathfrak{P}}:\left\{w_{0}, w_{1}, \ldots\right\} \mapsto w_{0} \wedge w_{1} \wedge w_{2} \wedge \cdots .
\end{aligned}
$$

При изменении репера $\left\{w_{0}, w_{1}, \ldots\right\}$, порождающего подпространство $w \subset \mathscr{H}$ действием (справа) элемента $g \in \mathfrak{G} \mathfrak{l}\left(\mathscr{H}_{+}\right)$, меняется только образ действия $\widehat{\mathfrak{P}}$ на ненулевой мультипликативный множитель $\operatorname{det}(g)$. Поэтому плюккерово отображение (2.12) на расслоении решетки проецируется в отображение вложения $\operatorname{Gr}_{\mathscr{H}_{+}}(\mathscr{H})$ в проективизацию $\mathbf{P}\left(\mathscr{F}_{0}\right)$ пространства $\mathscr{F}_{0}$

$$
\begin{aligned}
& \mathfrak{P}: \operatorname{Gr}_{\mathscr{H}_{+}}(\mathscr{H}) \rightarrow \mathbf{P}\left(\mathscr{F}_{0}\right), \\
& \mathfrak{P}:\left\{w_{0}, w_{1}, \ldots\right\} \mapsto\left[w_{0} \wedge w_{1} \wedge w_{2} \wedge \cdots\right],
\end{aligned}
$$

где $[|v\rangle]$ обозначает класс проективной эквивалентности элемента $|v\rangle \in \mathscr{F}_{0}$. В частности,

$$
\mathfrak{P}\left(\mathscr{H}_{\lambda}\right)=[|\lambda\rangle] .
$$

Образ $\operatorname{Gr}_{\mathscr{H}_{+}}(\mathscr{H})$ под действием $\mathfrak{P}$ является орбитой $\mathscr{H}_{+}$под действием компоненты единицы общей линейной группы $\mathfrak{G l}(\mathscr{H})$ (снова соответствующим образом определенной, как в [15] или [17]).

Плюккеровы координаты $\left\{\pi_{\lambda}(|v\rangle)\right\}$ элемента $|v\rangle \in \mathscr{F}_{0}$ есть просто его компоненты относительно ортонормированного базиса $\{|\lambda\rangle\}$ :

$$
\begin{aligned}
\pi_{\lambda}(|v\rangle) & =\langle\lambda \mid v\rangle, \\
|v\rangle & =\sum_{\lambda} \pi_{\lambda}|\lambda\rangle .
\end{aligned}
$$

При применении плюккерова отображения $\widehat{\mathfrak{P}}$ к элементу $\left\{w_{0}, w_{1}, \ldots\right\} \in$ $\operatorname{Fr}_{\mathscr{H}_{+}}(\mathscr{H})$, порождающему $w \in \operatorname{Gr}_{\mathscr{H}_{+}(\mathscr{H})}$, плюккеровы координаты его образа становятся однородными координатами образа $\mathfrak{P}(w)$ плюккерова отображения (2.13):

$$
\pi_{\lambda}(w):=\pi_{\lambda}(\mathfrak{P}(w)) .
$$


Из сказанного выше следует, что

$$
\pi_{\lambda}(w)=\operatorname{det}\left(W_{\lambda}\right)
$$

и поэтому базисы голоморфных сечений $\mathbf{H}^{0}\left(\operatorname{Gr}_{\mathscr{H}}(\mathscr{H}), \operatorname{Det}^{*}\right)$ paccлоения Det*, определенные разбиениями $\lambda$, соответствуют в точности плюккеровым координатам.

Плюккеровы соотношения являются бесконечным набором квадратичных соотношений, определяющих образ $\operatorname{Gr}_{\mathscr{H}_{+}}(\mathscr{H})$ под действием плюккерова отображения. Они следуют из того факта, что для любого $w \in \operatorname{Gr}_{\mathscr{H}_{+}}(\mathscr{H})$ образ $\mathfrak{P}(w)$ является разложимым элементом из $\mathscr{F}_{0}$. Плюккеровы координаты $\pi_{\lambda}(w)$, таким образом, не независимы; их можно выразить на открытом плотном аффинном подмногообразии как конечные детерминанты, зависящие от намного меньшего подмножества, состоящего, например, из тех плюккеровых координат, которые соответствуют разбиениям-крюкам. Удобно использовать обозначения Фробениуса $\left(a_{1}, a_{2}, \ldots, a_{r} \mid b_{1}, b_{2}, \ldots, b_{r}\right)$ для разбиения, [18], где $\left(a_{i}, b_{i}\right)$ - числа элементов справа и ниже $i$-го диагонального элемента диаграммы Юнга для $i=1, \ldots, r$. Разбиение-крюк $\lambda=\left(a+1,1^{b}\right)$ - это разбиение с $r=1$, в обозначениях Фробениуса, следовательно, выражающееся как $(a \mid b)$.

Чтобы понять, как плюккеровы координаты $\pi_{\left(a_{1}, a_{2}, \ldots, a_{r} \mid b_{1}, b_{2}, \ldots, b_{r}\right)}(w)$, соответствующие произвольному разбиению, выражаются в терминах координат разбиений-крюков $\left\{\left(a_{i} \mid b_{j}\right)\right\}_{1 \leqslant i, j \leqslant r}$, проще всего предположить, что $w$ входит в "большую клетку", в которой отображение $w_{+}: \mathscr{H}_{+} \rightarrow \mathscr{H}_{+}$обратимо. Тогда бесконечная матрица $W_{+}$из $(2.5)$ также обратима, и мы можем определить аффинные координаты как матричные элементы матрицы

$$
A:=W_{-} W_{+}^{-1} .
$$

По соглашению, индексы $(a, b)$ будут принимать неотрицательные целые значения, следовательно, покомпонентная интерпретация определения (2.18) есть

$$
A_{a b}:=\left(W_{-} W_{+}^{-1}\right)_{a b}, \quad a, b \in \mathbb{N} .
$$

Однородные координаты в этом базисе имеют вид

$$
W W_{+}^{-1}=\left(\begin{array}{l}
\mathbf{I} \\
A
\end{array}\right),
$$

где $\mathbf{I}$ есть полубесконечная единичная матрица, $I_{i j}=\delta_{-i-1, j}$, которая, ввиду соглашения по нумерации, имеет единичные $(i, j)$-е элементы для $i \leqslant-1, j \geqslant 0$, и соглашение по нумерации для матрицы $A$ в нижнем блоке состоит в том, что пара индексов $(a, b)$ начинается с $(0,0)$ в верхнем левом углу и возрастает вниз и вправо. Это позволяет нам выразить все плюккеровы координаты как конечные детерминанты, зависящие от аффинных координат на большой клетке.

ПРЕДЛОЖЕНИЕ 2.1. Плюккеровъ координатъь $\pi_{\left(a_{1}, a_{2}, \ldots, a_{r} \mid b_{1}, b_{2}, \ldots, b_{r}\right)}(w)$, соответствующие разбиению $\left(a_{1}, a_{2}, \ldots, a_{r} \mid b_{1}, b_{2}, \ldots, b_{r}\right)$, есть

$$
\begin{aligned}
\pi_{\left(a_{1}, a_{2}, \ldots, a_{r} \mid b_{1}, b_{2}, \ldots, b_{r}\right)}(w) & =\operatorname{det}\left(W_{\left(a_{1}, a_{2}, \ldots, a_{r} \mid b_{1}, b_{2}, \ldots, b_{r}\right)}\right) \\
& =\left.(-1)^{\sum_{k=1}^{r} b_{k}} \operatorname{det}\left(A_{a_{i} b_{j}}\right)\right|_{1 \leqslant i, j \leqslant r} \operatorname{det}\left(W_{+}\right) .
\end{aligned}
$$


В частности, мы можем рассмотреть случай разбиений-крюков, которые, согласно (2.21), совпадают с точностью до знака с компонентами матрицы аффинных координат $A$, позволяя всем остальным плюккеровым координатам быть выраженными как конечные детерминанты от них.

СлЕДСТвИЕ 2.1. Плюккеровъ координаты, соответствующие разбиениюкрюку $(a \mid b)$, есть, с точностью до знака, $(a, b)$-аффинные координаты,

$$
\pi_{(a \mid b)}(w)=(-1)^{b} A_{a b},
$$

и, следовательно,

$$
\pi_{\left(a_{1}, a_{2}, \ldots, a_{r} \mid b_{1}, b_{2}, \ldots, b_{r}\right)}(w)=\operatorname{det}\left(\left.\pi_{\left(a_{i} \mid b_{j}\right)}(w)\right|_{1 \leqslant i, j \leqslant r}\right) .
$$

2.2. Абелева группа потока $\Gamma_{+}$и $\tau$-функция КП. Теперь мы вводим абелеву подгруппу $\Gamma_{+} \subset \mathfrak{G l}(\mathscr{H})$, состоящую из ненулевых элементов $\mathscr{H}_{+}$, нормированных таким образом, чтобы равняться 1 в начале координат $z=0$ :

$$
\Gamma_{+}:=\left\{\gamma(\mathbf{t}):=\exp \left(\sum_{i=1}^{\infty} t_{i} z^{i}\right)\right\}, \quad \mathbf{t}:=\left(t_{1}, t_{2}, \ldots\right),
$$

действующую на $\mathscr{H}$ умножением

$$
\begin{aligned}
\Gamma_{+} \times \mathscr{H} & \rightarrow \mathscr{H} \\
(\gamma, f) & \mapsto \gamma f .
\end{aligned}
$$

Это индуцирует действие на грассманиане

$$
\begin{aligned}
\Gamma_{+} \times \operatorname{Gr}_{\mathscr{H}}(\mathscr{H}) & \rightarrow \operatorname{Gr}_{\mathscr{H}+}(\mathscr{H}), \\
(\gamma(\mathbf{t}), w) & \mapsto \gamma(\mathbf{t}) w,
\end{aligned}
$$

которое поднимается до действия на расслоении $\operatorname{Det}^{*} \rightarrow \operatorname{Gr}_{\mathscr{H}_{+}}(\mathscr{H})$ и определяет действие на пространстве голоморфных сечений $\mathrm{H}^{0}\left(\operatorname{Det}^{*}, \operatorname{Gr}_{\mathscr{H}_{+}}(\mathscr{H})\right)$

$$
\begin{gathered}
\Gamma_{+} \times \mathrm{H}^{0}\left(\operatorname{Det}^{*}, \operatorname{Gr}_{\mathscr{H}+}(\mathscr{H})\right) \rightarrow \mathrm{H}^{0}\left(\operatorname{Det}^{*}, \operatorname{Gr}_{\mathscr{H}_{+}}(\mathscr{H})\right) \\
(\gamma(\mathbf{t}), \sigma) \mapsto \widetilde{\gamma}(\mathbf{t}) \sigma:=\sigma \circ \gamma^{-1}(\mathbf{t}) \\
\widetilde{\gamma}(\mathbf{t}) \sigma(w):=\widetilde{\gamma}(\mathbf{t}) \sigma\left(\gamma^{-1}(\mathbf{t}) w\right) .
\end{gathered}
$$

Последнее совпадает, с точностью до нормировки, с индуцированным действием на $\mathscr{F}_{0} \subset \Lambda \mathscr{H}$. Пусть

$$
w(\mathbf{t})=\gamma(\mathbf{t})(w)
$$

- образ $w \in \operatorname{Gr}_{\mathscr{H}_{+}}(\mathscr{H})$ под действием элемента $\gamma(\mathbf{t})$ группы, и пусть $W(\mathbf{t})$ ее матрица однородных координат относительно стандартного базиса мономов $\left\{e_{j}\right\}_{j \in \mathbb{Z}}$. Тогда

$$
\pi_{\lambda}(w(\mathbf{t}))=\operatorname{det}\left(W_{\lambda}(\mathbf{t})\right)
$$

- плюккеровы координаты элемента $w(\mathbf{t})$, соответствующие разбиению $\lambda$. $\tau$-функция КП определяется как плюккерова координата, соответствующая тривиальному разбиению $\lambda=0$ :

$$
\tau_{w}(\mathbf{t}):=\pi_{0}(w(\mathbf{t}))=\operatorname{det}\left(W_{+}(\mathbf{t})\right)
$$


Поскольку, как будет показано в следующем п. 2.3, все остальные плюккеровы координаты образа $w(\mathbf{t})$ могут быть определены из $\tau_{w}(\mathbf{t})$ применением дифференциальных операторов с постоянными коэффициентами по переменной $\mathbf{t}$, определенных в терминах функций Шура, то плюккеровы соотношения для $\mathfrak{P}(w(\mathbf{t}))$ могут быть выражены как бесконечная система билинейных дифференциальных соотношений, которым удовлетворяет $\tau_{w}(\mathbf{t})$ - соотношений Хироты [15], которые эквивалентны уравнениям потока иерархии КП.

2.3. Разложение в ряд по функциям Шура. Напомним, что если мы рассмотрим параметры потока $\mathbf{t}=\left(t_{1}, t_{2}, \ldots\right)$ как степенные суммы в терминах набора $\left\{x_{1}, \ldots, x_{N}\right\}$ из $N$ вспомогательных переменных

$$
t_{i}=\frac{1}{i} \sum_{a=1}^{N} x_{a}^{i}
$$

то функция Шура $s_{\lambda}$, являющаяся неприводимым характером тензорного представления $\mathfrak{G l}(N)$ с типом симметрий, соответствующим разбиению $\lambda$, задается формулой Якоби-Труди [18]

$$
s_{\lambda}(\mathbf{t})=\left.\operatorname{det}\left(h_{\lambda_{i}-i+j}\right)\right|_{1 \leqslant i, j \leqslant n}
$$

для любого $n \geqslant \ell(\lambda)$, где $\left\{h_{j}(\mathbf{t})\right\}_{j=1, \ldots, \infty}-$ полные симметричные функции, определенные формулой для порождающей функции

$$
\exp \left(\sum_{i=1}^{\infty} t_{i} z^{i}\right)=\sum_{j=0}^{\infty} h_{j}(\mathbf{t}) z^{j} .
$$

Имеем $h_{0}(\mathbf{t})=1$, и по $(2.32)$ понятно, что $h_{j}(\mathbf{t}):=0$ для $j<0$.

Пусть дана функция $f(\mathbf{t})$, допускающая разложение в ряд Тейлора по переменным потока в начале координат $0:=(0,0, \ldots)$,

$$
f(\mathbf{t})=\left.\left(\exp \left(\sum_{i=1}^{\infty} t_{i} \frac{\partial}{\partial \tilde{t}_{i}}\right) f(\tilde{\mathbf{t}})\right)\right|_{\tilde{\mathbf{t}}=\mathbf{0}},
$$

где $\tilde{\mathbf{t}}=\left(\tilde{t}_{1}, \tilde{t}_{2}, \ldots\right)$. Тогда мы можем использовать функции Шура как базис и представить ряд в виде

$$
f(\mathbf{t})=\sum_{\lambda} f_{\lambda} s_{\lambda}(\mathbf{t})
$$

(Это определяет “эквивалентность Бозе-Ферми”, которая ассоциирует элемент $\sum_{\lambda} f_{\lambda}|\lambda\rangle$ фермионного фоковского пространства $\mathscr{F}_{0}$ элементу $f$ пространства Бозе-Фока, рассматриваемого как симметричная функция стоящей за ней бесконечной последовательности параметров $\left\{x_{a}\right\}_{a=1, \ldots, \infty}$, связанных с помощью (2.31), в индуктивном пределе, с параметрами потока t.) Используя тождество Коши-Литтлвуда [18]

$$
\exp \left(\sum_{i=1}^{\infty} i t_{i} \tilde{t}_{i}\right)=\sum_{\lambda} s_{\lambda}(\mathbf{t}) s_{\lambda}(\tilde{\mathbf{t}})
$$


в виде

$$
\exp \left(\sum_{i=1}^{\infty} t_{i} \frac{\partial}{\partial t_{i}}\right)=\sum_{\lambda} s_{\lambda}(\mathbf{t}) s_{\lambda}\left(\partial_{\mathbf{t}}\right)
$$

где

мы получаем

$$
\partial_{\mathbf{t}}:=\left\{\frac{1}{i} \frac{\partial}{\partial t_{i}}\right\}_{i=1,2, \ldots},
$$

$$
f_{\lambda}=\left.s_{\lambda}\left(\partial_{\mathbf{t}}\right)(f(\mathbf{t}))\right|_{\mathbf{t}=\mathbf{0}} .
$$

Для $\tau$-функции $\tau_{w}(\mathbf{t})$, коэффициенты этого разложения совпадают с плюккеровыми координатами $\pi_{\lambda}(w)$ исходной точки $w \in \operatorname{Gr}_{\mathscr{H}_{+}}(\mathscr{H})$.

Предложение 2.2 (М. и Я. Сато [15]). Разложение по функииям Шура функиии $\tau_{w}(\mathbf{t})$ имеет вид

$$
\tau_{w}(\mathbf{t})=\sum_{\lambda} \pi_{\lambda}(w) s_{\lambda}(\mathbf{t})
$$

Следовательно, плюккеровы координаты задаются как

$$
\pi_{\lambda}(w)=\left.s_{\lambda}\left(\partial_{\mathbf{t}}\right)\left(\tau_{w}(\mathbf{t})\right)\right|_{\mathbf{t}=\mathbf{0}} .
$$

ДокАЗАТЕЛЬство. Используя формулу (2.33), легко показать, что матричное представление действия (2.25) имеет вид

$$
W(\mathbf{t})=\left(\begin{array}{cc}
H_{++}(\mathbf{t}) & H_{+-}(\mathbf{t}) \\
0 & H_{--}^{T}(\mathbf{t})
\end{array}\right)\left(\begin{array}{l}
W_{+} \\
W_{-}
\end{array}\right),
$$

где

$$
\begin{aligned}
H_{++}(\mathbf{t}):=\left(\begin{array}{cccc}
\ddots & \ddots & \vdots & \vdots \\
\ddots & 1 & h_{1} & h_{2} \\
\cdots & 0 & 1 & h_{1} \\
\cdots & 0 & 0 & 1
\end{array}\right), \\
H_{+-}(\mathbf{t}):=\left(\begin{array}{ccccc}
\vdots & & \\
h_{3} & \vdots & \\
h_{2} & h_{3} & \vdots \\
h_{1} & h_{2} & h_{3} & \ldots
\end{array}\right), H_{--}(\mathbf{t}):=\left(\begin{array}{ccccc}
1 & h_{1} & h_{2} & h_{3} & \cdots \\
0 & 1 & h_{1} & h_{2} & \cdots \\
0 & 0 & 1 & h_{1} & \cdots \\
\vdots & \vdots & \vdots & \ddots & \ddots
\end{array}\right) .
\end{aligned}
$$

Полагая

$$
H(\mathbf{t}):=\left(H_{++}(\mathbf{t}) \quad H_{+-}(\mathbf{t})\right)
$$

имеем

$$
\tau_{w}(\mathbf{t})=\operatorname{det}(H(\mathbf{t}) W)=\sum_{\lambda} \operatorname{det}\left(H_{\lambda}(\mathbf{t})\right) \operatorname{det}\left(W_{\lambda}\right)=\sum_{\lambda} \pi_{\lambda}\left(H^{T}(\mathbf{t})\right) \pi_{\lambda}(w),
$$

где второе равенство есть тождество Бине-Коши, и

$$
\pi_{\lambda}(H(\mathbf{t})):=\left.\operatorname{det}\left(h_{\lambda_{i}-i+j}(\mathbf{t})\right)\right|_{1 \leqslant i, j \leqslant n}=s_{\lambda}(\mathbf{t})
$$


согласно (2.32). Мы, таким образом, получаем разложение по функциям Шура $(2.40) \tau$-функции.

На “большой клетке" каждое $\pi_{\lambda}(w)$ определяется посредством (2.21) как конечный детерминант от аффинных координат $\left\{A_{a b}\right\}$ точки $w$, которые, по (2.22), совпадают с точностью до знака с плюккеровыми координатами разбиения-крюка. Следовательно, нам нужно только применить (2.41), чтобы получить

$$
(-1)^{b} A_{a b}=\pi_{(a \mid b)}(w)=\left.s_{(a \mid b)}\left(\partial_{\mathbf{t}}\right)\left(\tau_{w}(\mathbf{t})\right)\right|_{\mathbf{t}=\mathbf{0}} .
$$

Подставляя выражение $(2.21)$ для плюккеровых координат $\pi_{\left(a_{1}, \ldots, a_{r} \mid b_{1}, \ldots, b_{r}\right)}$ в (2.40), мы получаем следующий результат.

СЛЕДСТвиЕ 2.2 .

$$
\frac{\tau_{w}(\mathbf{t})}{\tau_{w}(\mathbf{0})}=\left.\sum_{\lambda}(-1)^{\sum_{k=1}^{r} b_{k}} \operatorname{det}\left(A_{a_{i} b_{j}}\right)\right|_{1 \leqslant i, j \leqslant r} s_{\lambda}(\mathbf{t}) .
$$

Дальнейшее упрощение может быть получено с использованием следующего тождества, простое доказательство которого следует из изложенных выше определений.

Лемма 2.1. Справедливо тождество

$$
s_{(a \mid b)}(\mathbf{t})=(-1)^{b} \sum_{j=1}^{b+1} h_{b-j+1}(-\mathbf{t}) h_{a+j}(\mathbf{t}) .
$$

ДокАзАтЕльство. Согласно формуле Якоби-Труди (2.32),

$$
s_{(a \mid b)}(\mathbf{t})=\operatorname{det}\left(\begin{array}{cc}
\mathbf{h}^{T} & h \\
\mathbf{H} & \mathbf{k}
\end{array}\right),
$$

где

$$
\begin{gathered}
\mathbf{h}^{T}=\left(h_{a+1}(\mathbf{t}), h_{a+2}(\mathbf{t}), \ldots, h_{a+b}(\mathbf{t})\right), \\
\mathbf{k}:=\left(\begin{array}{c}
h_{b}(\mathbf{t}) \\
h_{b-1}(\mathbf{t}) \\
\vdots \\
h_{1}(\mathbf{t})
\end{array}\right), \quad \mathbf{H}:=\left(\begin{array}{cccccc}
1 & h_{1}(\mathbf{t}) & h_{2}(\mathbf{t}) & \ldots & \ldots & h_{b-1}(\mathbf{t}) \\
0 & 1 & h_{1}(\mathbf{t}) & h_{2}(\mathbf{t}) & \cdots & h_{b-2}(\mathbf{t}) \\
0 & 0 & \ddots & \ddots & \ddots & \vdots \\
\vdots & \vdots & & & & \vdots \\
0 & 0 & \ldots & & \ldots & 1
\end{array}\right) .
\end{gathered}
$$

Из формулы для производящей функции (2.33) следует, что обратная матрица $\mathbf{H}^{-1}$ задана как

$$
\mathbf{H}^{-1}:=\left(\begin{array}{cccccc}
1 & h_{1}(-\mathbf{t}) & h_{2}(-\mathbf{t}) & \cdots & \cdots & h_{b-1}(-\mathbf{t}) \\
0 & 1 & h_{1}(-\mathbf{t}) & h_{2}(-\mathbf{t}) & \cdots & h_{b-2}(-\mathbf{t}) \\
0 & 0 & \ddots & \ddots & \ddots & \vdots \\
\vdots & \vdots & & & & \vdots \\
0 & 0 & \cdots & & \cdots & 1
\end{array}\right)
$$


И

$$
\sum_{j=-a}^{b} h_{a+j}(-\mathbf{t}) h_{b-j}(\mathbf{t})=\delta_{a b}
$$

Детерминант матрицы

$$
\left(\begin{array}{cc}
\mathbf{0}^{T} & \mathbf{H}^{-1} \\
1 & -h^{-1} \mathbf{h}^{T} \mathbf{H}^{-1}
\end{array}\right)
$$

равен $(-1)^{b}$, а ее произведение на матрицу из (2.51) есть

$$
\left(\begin{array}{cc}
\mathbf{h}^{T} & h \\
\mathbf{H} & \mathbf{k}
\end{array}\right)\left(\begin{array}{cc}
\mathbf{0}^{T} & \mathbf{H}^{-1} \\
1 & -h^{-1} \mathbf{h}^{T} \mathbf{H}^{-1}
\end{array}\right)=\left(\begin{array}{cc}
h & \mathbf{0}^{T} \\
\mathbf{k} & \mathbf{I}-h^{-1} \mathbf{k h}^{T} \mathbf{H}^{-1}
\end{array}\right) .
$$

Поэтому

$$
s_{(a \mid b)}(\mathbf{t})=(-1)^{b} h \operatorname{det}\left(\mathbf{I}-h^{-1} \mathbf{k h}^{T} \mathbf{H}^{-1}\right)=(-1)^{b}\left(h-\mathbf{H}^{-1} \mathbf{k h}^{T}\right) .
$$

Но из (2.54) и (2.55) следует, что

$$
\mathbf{H}^{-1} \mathbf{k}=-\left(\begin{array}{c}
h_{b}(-\mathbf{t}) \\
h_{b-1}(-\mathbf{t}) \\
\vdots \\
h_{1}(-\mathbf{t})
\end{array}\right)
$$

откуда, ввиду определения (2.52) для $h$, следует (2.50), что и требовалось доказать.

Подстановка равенства (2.50) в (2.48), таким образом, дает следующее утверждение.

СлЕДСТВиЕ 2.3 .

$$
A_{a b}=-\left.\sum_{j=0}^{b} h_{a+j-1}\left(-\partial_{\mathbf{t}}\right) h_{b-j}\left(\partial_{\mathbf{t}}\right)\left(\tau_{w}(\mathbf{t})\right)\right|_{\mathbf{t}=0} .
$$

Соотношения $(2.21),(2.41),(2.48)$, определяющие плюккеровы координаты элемента $w \in \operatorname{Gr}_{\mathscr{H}_{+}}(\mathscr{H})$ на "большой клетке", эквивалентны результату, что формальная функция Бейкера-Ахиезера [13], [14], [17], определенная формулой Сато [15]

$$
\psi_{w}(z, \mathbf{t})=\exp \left(\sum_{i=1}^{\infty} t_{i} z^{i}\right) \frac{\tau_{w}\left(\mathbf{t}-\left[z^{-1}\right]\right)}{\tau_{w}(\mathbf{t})}
$$

где

$$
\left[z^{-1}\right]:=\left(\frac{1}{z}, \frac{1}{2 z^{2}}, \frac{1}{3 z^{3}}, \ldots\right),
$$

принимает значения в $w \in \operatorname{Gr}_{\mathscr{H}_{+}}(\mathscr{H})$ для всех значений $\mathbf{t}$.

Следуя Сато, мы можем также ввести дуальную функцию Бейкера:

$$
\Psi_{w}^{*}(z, \mathbf{t})=-\frac{\tau_{w}\left(\mathbf{t}+\left[z^{-1}\right]\right)}{\tau(\mathbf{t})} \exp \left\{-\sum_{i=1}^{\infty} t_{i} z^{i}\right\} .
$$

Тогда, как показано в [13]-[15], уравнения иерархии КП могут все быть выражены в виде билинейных уравнений Хироты на $\tau$-функцию. 
ТЕОРемА 2.1 (билинейное соотношение Хироты [13]-[15]).

$$
\operatorname{Res}_{z=0} \Psi_{z}^{*}(z, \mathbf{t}) \Psi_{z}^{*}(z, \tilde{\mathbf{t}}) \equiv 0
$$

где $\operatorname{Res}_{z=0}$ обозначает коэффициент при $z^{-1}$ в формальном разложении Лорана в $z=0$, и соотношение удовлетворяется тождественно на бесконечном наборе переменных потока КП $\mathbf{t}=\left(t_{1}, t_{2}, \ldots\right), \tilde{\mathbf{t}}=\left(\tilde{t}_{1}, \tilde{t}_{2}, \ldots\right)$.

ЗАмечАниЕ 2.3. Ввиду (2.63), соотношение (2.64) записывается эквивалентным образом как

$$
\operatorname{Res}_{z=0} \exp \left\{\sum_{i=1}^{\infty} t_{i} z^{i}\right\} \exp \left\{-\sum_{i=1}^{\infty} \tilde{t}_{i} z^{i}\right\} \tau\left(\mathbf{t}-\left[z^{-1}\right]\right) \tau\left(\tilde{\mathbf{t}}+\left[z^{-1}\right]\right) \equiv 0
$$

тождественно по $\mathbf{t}$ и $\tilde{\mathbf{t}}$.

ЗАмечАниЕ 2.4. В [13]-[15] показано также, что (2.61) есть просто выражение от бесконечного набора плюккеровых соотношений, которым удовлетворяют коэффициенты $\pi_{\lambda}(w)$, возникающие в разложении по функциям Шуpa $(2.40)$.

\section{3. Алгебраические кривые}

3.1. Функция Бейкера-Ахиезера и $\tau$-функция для алгебраических кривых. Особенно важный класс $\tau$-функций состоит из функций, ассоциированных с алгебраическими кривыми [12], [11], [16]. Для их определения необходимы следующие данные: алгебраическая кривая $X$ рода $g$, положительный неспециальный дивизор степени $g$

$$
\mathscr{D}:=\sum_{i=1}^{g} p_{i}, \quad p_{i} \in X,
$$

(или, что эквивалентно, положительное линейное расслоение $\mathscr{L} \rightarrow X$ степени $g$ в общем положении, удовлетворяющее подходящим общим условиям стабильности), точка "на бесконечности" $p_{\infty} \in X$ и локальный параметр $\xi=1 / z$, определенный на диске

$$
D_{\infty}:=\left\{p(\zeta),|\zeta| \leqslant 1, p(0)=p_{\infty}\right\}
$$

с центром в $p_{\infty}$. Точки $p_{i}$ предполагаются лежащими в дополнении $D_{0}:=$ $X-D_{\infty}$. Отождествляя $S^{1}$ с $\partial D_{\infty}$, получаем, что ассоциированный элемент $w:=w\left(X, \mathscr{D}, p_{\infty}, \zeta\right) \in \operatorname{Gr}_{\mathscr{H}_{+}}(\mathscr{H})$ есть замыкание пространства функций $f \in$ $L^{2}\left(S^{1}\right)$, допускающих мероморфное продолжение на $\bar{D}_{0}$ с дивизором полюсов, подчиненным $\mathscr{D}$.

Для реализации построения мы используем каноническую $\theta$-функцию от $g$ переменных, $g \geqslant 1$ :

$$
\theta(\mathbf{z})=\sum_{\mathbf{m} \in \mathbb{Z}^{g}} \exp \left\{\imath \pi \mathbf{m}^{T} \mathbf{T} \mathbf{m}+2 \imath \pi \mathbf{m}^{T} \mathbf{z}\right\}, \quad \mathbf{z} \in \mathbb{C}^{g},
$$


где $\mathbf{T}$ есть комплексная симметричная $(g \times g)$-матрица с положительно определенной мнимой частью. Пространство таких матриц (верхняя полуплоскость Зигеля) будет обозначаться $\mathscr{S}^{g} . \theta$-функция голоморфна на $\mathbb{C}^{g} \times \mathscr{S}^{g}$ и удовлетворяет

$$
\theta(\mathbf{z}+\mathbf{n})=\theta(\mathbf{z}), \quad \theta(\mathbf{z}+\mathbf{T n})=\exp \left\{-\imath \pi\left(\mathbf{n}^{T} \mathbf{T n}+2 \mathbf{z}^{T} \mathbf{n}\right)\right\} \theta(\mathbf{z}) .
$$

В рассмотренном случае $\theta$-функций, ассоциированных с алгебраической кривой, имеем

$$
\mathbf{T}:=\mathfrak{A}^{-1} \mathfrak{B},
$$

где $\mathfrak{A}$ и $\mathfrak{B}$ - матрицы периодов базиса голоморфных дифференциалов.

В [11], [12], [16] показано, что соответствующая функция Бейкера-Ахиезера может быть выбрана как ограничение на $\partial D_{+}$мероморфной функции на $X-p_{\infty}$ с дивизором полюсов $\mathscr{D}$, имеющей существенную особенность в $p_{\infty}$, вида

$$
\psi_{w}(p(\zeta), \mathbf{t}) \sim \exp \left\{\sum_{i=1}^{\infty} t_{i} z^{i}\right\}\left(1+\mathscr{O}\left(\frac{1}{z}\right)\right) .
$$

Из теоремы Римана-Роха следует, что такие функции образуют только одномерное пространство. Они могут быть выражены, с точностью до нормировки, зависящей от $\mathbf{t}$, как

$$
\widetilde{\psi}_{w}(p, \mathbf{t})=\exp \left\{\int_{p_{0}}^{p} \Omega(\mathbf{t})\right\} \frac{\theta\left(\mathscr{A}(p)-\mathscr{A}(\mathscr{D})+\sum_{i=1}^{\infty} \mathbf{U}_{i} t_{i}-\mathbf{K}\right)}{\theta(\mathscr{A}(p)-\mathscr{A}(\mathscr{D})-\mathbf{K})},
$$

где риманова матрица периодов $\mathbf{T}$ лигонизации, полученной разрезанием вдоль канонического базиса гомологий $\left(\mathfrak{a}_{1}, \ldots, \mathfrak{a}_{g} ; \mathfrak{b}_{1}, \ldots, \mathfrak{b}_{g}\right)$ с матрицей пересечений

$$
\mathfrak{a}_{i} \circ \mathfrak{a}_{j}=\mathfrak{b}_{i} \circ \mathfrak{b}_{j}=0, \quad \mathfrak{a}_{i} \circ \mathfrak{b}_{j}=\delta_{i j},
$$

$p_{0}$ есть произвольно выбранная базисная точка,

$$
\begin{array}{ll}
\mathscr{A}: & \mathscr{S}^{g}(X) \rightarrow \mathbb{C}^{g}, \\
\mathscr{A}: \quad \sum_{j=1}^{g} p_{j} \mapsto \sum_{j=1}^{g} \int_{p_{0}}^{p_{j}} \boldsymbol{\omega}, \quad \boldsymbol{\omega}=\left(\begin{array}{c}
\omega_{1} \\
\vdots \\
\omega_{g}
\end{array}\right),
\end{array}
$$

есть отображение Абеля с $i$-й компонентой

$$
\mathscr{A}_{i}:=\sum_{j=1}^{g} \int_{p_{0}}^{p_{j}} \omega_{i}, \quad i=1, \ldots, g
$$

где $\left(\omega_{1}, \ldots, \omega_{g}\right)$ есть канонически нормированный базис пространства $\mathrm{H}^{0}(K)$ голоморфных абелевых дифференциалов

$$
\oint_{\mathfrak{a}_{i}} \omega_{j}=\delta_{i j}, \quad \oint_{\mathfrak{b}_{i}} \omega_{j}=\mathrm{T}_{i j}
$$


и $\mathbf{K} \in \mathbb{C}^{g}$ - риманова константа, выбранная таким образом, что $\theta(\mathscr{A}(p)-$ $\mathscr{A}(\mathscr{D})-\mathbf{K})$ обращается в нуль в $g$ точках $p=p_{i}$ дивизора $\mathscr{D}$.

Определим линейное семейство абелевых дифференциалов второго рода

$$
\Omega(\mathbf{t})=\sum_{j=1}^{\infty} \Omega_{j} t_{j}
$$

где $\Omega_{i}$ есть единственный нормированный абелев дифференциал второго рода с дивизором полюсов степени $j+1$ в $p_{\infty}$, имеющий локальный вид

$$
\Omega_{j} \sim d\left(z^{j}\right)+(\text { голоморфный) }
$$

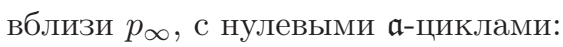

$$
\oint_{\mathfrak{a}_{i}} \Omega_{j}=0, \quad i=1, \ldots, g .
$$

Тогда $2 \pi \imath \mathbf{U}_{j} \in \mathbb{C}^{g}$ определяется как его вектор $\mathfrak{b}$-циклов с компонентами

$$
\oint_{\mathfrak{b}_{k}} \Omega_{j}=: 2 \pi \imath\left(\mathbf{U}_{j}\right)_{k}, \quad k=1, \ldots, g .
$$

Для сравнения с формальной функцией Бейкера $\psi_{a}(z, \mathbf{t})$, возникающей в формуле Сато (2.61), нужно интерпретировать функцию $p=p(z)$ внутри проколотого диска $D_{\infty}-p_{\infty}$ и на его границе и нормировать $\widetilde{\psi}(p, \mathbf{t})$ в формуле (3.7) таким образом, чтобы получить правильное локальное разложение (3.6) вблизи $\xi=0$ :

$$
\psi_{w}(p(\xi), \mathbf{t})=\frac{\widetilde{\psi}_{w}(p(\xi), \mathbf{t})}{a_{0}(\mathbf{t})}
$$

где

$$
\widetilde{\psi}_{w}(p(\xi), \mathbf{t}) \sim \exp \left\{\int_{p_{0}}^{p(\xi)} \Omega(\mathbf{t})\right\}\left(a_{0}(\mathbf{t})+a_{1}(\mathbf{t}) \xi+\cdots\right) .
$$

Так как $\int_{p_{0}}^{p} \Omega_{i}$ имеет локально разложение

$$
\int_{p_{0}}^{p} \Omega_{i}=\xi^{-i}+\sum_{j=1}^{\infty} \frac{1}{j} Q_{i j} \xi^{j}+q_{j}
$$

где

$$
Q_{i j}=Q_{j i}, \quad 1 \leqslant i, j \leqslant \infty,
$$

и $\mathscr{A}(p(z))$ имеет разложение (см. [12], [35])

$$
\mathscr{A}(p(z))=\mathscr{A}\left(p_{\infty}\right)-\sum_{j=1}^{\infty} \frac{1}{j} \mathbf{U}_{j} z^{-j},
$$

то получаем формулу

$$
\begin{aligned}
\psi_{w}(p(z), \mathbf{t})= & \exp \left\{\sum_{i=1}^{\infty} t_{i}\left(z^{i}+\sum_{j=1}^{\infty} \frac{1}{j} Q_{j i} z^{-j}\right)\right\} \\
& \times \frac{\theta\left(\mathbf{e}+\sum_{i=1}^{\infty} \mathbf{U}_{i}\left(t_{i}-z^{-i} / i\right)\right) \theta(\mathbf{e})}{\theta\left(\mathbf{e}-\sum_{i=1}^{\infty} \mathbf{U}_{i} z^{-i} / i\right) \theta\left(\mathbf{e}+\sum_{i=1}^{\infty} \mathbf{U}_{i} t_{i}\right)}
\end{aligned}
$$


вблизи $z=\infty$, где

$$
\mathbf{e}:=\mathscr{A}\left(p_{\infty}\right)-\mathscr{A}(\mathscr{D})-\mathbf{K}
$$

(Заметим, что из предположений, сделанных при выводе формулы (3.7), следует, что е не лежит в $\theta$-дивизоре; $\theta(\mathbf{e}) \neq 0$.) Заметим, что отношение $\theta$-функциональных множителей в (3.21),

$$
\frac{\theta(\mathbf{e})}{\theta\left(\mathbf{e}+\sum_{i=1}^{\infty} \mathbf{U}_{i} t_{i}\right)},
$$

не зависит от $z$, и, следовательно, пространство, порожденное значениями $\psi_{w}(p(z), \mathbf{t})$, совпадает с порожденным значениями

$$
\check{\psi}_{w}(p(z), \mathbf{t}):=\exp \left\{\sum_{i=1}^{\infty} t_{i}\left(z^{i}+\sum_{j=1}^{\infty} \frac{1}{j} Q_{j i} z^{-j}\right)\right\} \frac{\theta\left(\mathbf{e}+\sum_{i=1}^{\infty} \mathbf{U}_{i}\left(t_{i}-z^{-i} / i\right)\right)}{\theta\left(\mathbf{e}-\sum_{i=1}^{\infty} \mathbf{U}_{i} z^{-i} / i\right)} .
$$

Мы можем разложить оставшееся отношение $\theta$-функциональных выражений в степенные ряды по $z^{-1}$ :

$$
\begin{gathered}
\theta\left(\mathbf{e}+\sum_{i=1}^{\infty} \mathbf{U}_{i}\left(t_{i}-\frac{1}{i} z^{-i}\right)\right) \exp \left\{\sum_{i=1}^{\infty}-\frac{1}{i z^{i}} \frac{\partial}{\partial t_{i}}\right\}\left(\theta\left(\mathbf{e}+\sum_{i=1}^{\infty} \mathbf{U}_{i} t_{i}\right)\right) \\
=\sum_{j=0}^{\infty} z^{-j} h_{j}\left(-\nabla_{\mathbf{U}}\right) \theta\left(\mathbf{e}+\sum_{i=1}^{\infty} \mathbf{U}_{i} t_{i}\right)
\end{gathered}
$$

И

$$
\theta\left(\mathbf{e}-\sum_{i=1}^{\infty} \frac{1}{i} \mathbf{U}_{i} z^{-i}\right)=\sum_{j=0}^{\infty} z^{-j} h_{j}\left(-\nabla_{\mathbf{U}}\right) \theta(\mathbf{e})
$$

где

$$
\nabla_{\mathbf{U}}:=\left(\nabla_{\mathbf{U}_{1}}, \frac{1}{2} \nabla_{\mathbf{U}_{2}}, \frac{1}{3} \nabla_{\mathbf{U}_{3}}, \ldots\right)
$$

и $\nabla_{\mathbf{U}_{i}}$ есть производная по направлению в $\mathbb{C}^{g}$ вдоль $\mathbf{U}_{i}$.

Теперь определим базис $\left\{w_{0}, w_{1}, \ldots\right\}$ для $w$ формулами

$$
w_{0}(z):=\left.\check{\psi}(z, \mathbf{t})\right|_{\mathbf{t}=\mathbf{0}}=1, \quad w_{j}(z):=\left.\frac{\partial \check{\psi}(z, \mathbf{t})}{\partial t_{j}}\right|_{\mathbf{t}=\mathbf{0}}, \quad j \geqslant 1 .
$$

Тогда

$$
w_{j}(z)=z^{j}+P_{0 j}+\sum_{i=1}^{\infty}\left(\frac{1}{i} Q_{i j}+P_{i j}\right) z^{-i}, \quad j=1,2, \ldots,
$$

где

$$
\sum_{i=0}^{\infty} P_{i j} z^{-i}:=\frac{\sum_{i=0}^{\infty} M_{i j} z^{-i}}{\sum_{i=0}^{\infty} N_{i} z^{-i}}
$$

И

$$
M_{i j}:=\nabla_{\mathbf{U}_{j}} h_{i}\left(-\nabla_{\mathbf{U}}\right) \theta(\mathbf{e}), \quad N_{i}:=h_{i}\left(-\nabla_{\mathbf{U}}\right) \theta(\mathbf{e}) .
$$


Следовательно, аффинные координаты $A_{i j}$ элемента $w$ есть

$$
\begin{aligned}
A_{i j}:=\frac{1}{i+1} Q_{i+1, j}+P_{i+1, j}, & & i=0,1,2, \ldots, \quad j=1,2, \ldots, \\
A_{i 0}=0, & i & =0,1,2 \ldots .
\end{aligned}
$$

По следствию 2.1, это определяет плюккеровы координаты для всех разбиений-крюков и, значит, согласно (2.23), для всех разбиений.

Сравнивая формулу (3.21) для функции Бейкера с формулой Сато (2.61), мы видим что $\tau$-функция для $w=w\left(X, \mathscr{D}, p_{\infty}, \zeta\right)$ задается формулой (cp. [35])

$$
\tau_{w}(\mathbf{t})=\exp \left\{\sum_{i=1}^{\infty} \lambda_{i} t_{i}\right\} \exp \left\{-\frac{1}{2} \sum_{i, j=1}^{\infty} Q_{i j} t_{i} t_{j}\right\} \theta\left(\mathbf{e}+\sum_{i=1}^{\infty} t_{i} \mathbf{U}_{i}\right)
$$

где

$$
\lambda_{i}:=\mu_{i}+i \sum_{k=0}^{i-1} \frac{Q_{k, i-k}}{2 k(i-k)}
$$

с $\mu_{i}$, определенными по формуле

$$
\theta(\mathbf{e}) \exp \left\{\sum_{i=1}^{\infty} \frac{\mu_{i}}{i z^{i}}\right\}:=\theta\left(\mathbf{e}-\sum_{i=1}^{\infty} \frac{\mathbf{U}_{i}}{i z^{i}}\right)=\sum_{i=0}^{\infty} N_{i} z^{-i}
$$

С точки зрения иерархии КП, тем не менее, линейный экспоненциальный множитель $\exp \left\{\sum_{i=1}^{\infty} \lambda_{i} t_{i}\right\}$ в (3.32) может быть опущен, поскольку это в точности соответствует калибровочному преобразованию функции Бейкера-Ахиезеpa, которое постоянно по переменным t:

$$
\psi_{w}(z, \mathbf{t}) \rightarrow k(z) \psi_{w}(z, \mathbf{t}), \quad k(z):=\exp \left\{\sum_{i=1}^{\infty} \frac{\lambda_{i}}{i z^{i}}\right\}
$$

и оставляет решения уравнений потока КП инвариантными. Это равносильно замене $w \in \operatorname{Gr}_{\mathscr{H}_{+}}(\mathscr{H})$ на

$$
w_{k}:=\operatorname{span}\{k v, v \in w\} .
$$

Поэтому $\tau$-функция может быть задана в более простом калибровочно эквивалентном виде (ср. [16], [29], [35])

$$
\tau_{w}(\mathbf{t})=\exp \left\{-\frac{1}{2} \sum_{i, j=1}^{\infty} Q_{i j} t_{i} t_{j}\right\} \theta\left(\mathbf{e}+\sum_{i=1}^{\infty} t_{i} \mathbf{U}_{i}\right) .
$$

Впредь мы используем для этой $\tau$-функции обозначение $\tau(\mathbf{e}, \mathbf{t})=\tau_{w}(\mathbf{t})$.

ЗАмЕчАНИЕ 3.1. Несмотря на то что этот калибровочный множитель не воздействует на решения иерархии КП, он играет важную роль в более общей постановке неизоспектральных деформаций. Он связан с тензорным весом $\tau$-функции, рассмотренным как сечение линейного расслоения на пространстве модулей. (См. [36]--[38].) 
Применение формулы (2.60) напрямую к $\tau_{w}(\mathbf{t})$, определенной в $(3.37)$, дает альтернативный способ вычисления аффинных координат $A_{a b}$, который эквивалентен (3.31) с точностью до калибровочного преобразования.

Билинейные соотношения Хироты (2.65), определяющие иерархию КП, могут быть в этом случае эквивалентным образом записаны как

$$
\begin{aligned}
\operatorname{Res}_{\xi=0} \frac{1}{\xi^{2}}[\exp & \left\{\sum_{n=1}^{\infty} t_{n} \xi^{-n}\right\} \exp \left\{-\sum_{n=1}^{\infty} \frac{\xi^{n}}{n} \frac{\partial}{\partial t_{n}}\right\} \tau(\mathbf{e} ; \mathbf{t}) \\
& \left.\times \exp \left\{-\sum_{n=1}^{\infty} \tilde{t}_{n} \xi^{-n}\right\} \exp \left\{\sum_{n=1}^{\infty} \frac{\xi^{n}}{n} \frac{\partial}{\partial \tilde{t}_{n}}\right\} \tau(\mathbf{e} ; \tilde{\mathbf{t}})\right]=0
\end{aligned}
$$

где $\xi=\xi(q)$ - локальные координаты точки $q$ около $p, \xi(p)=0$.

3.2. Последовательности пробелов Вейерштрасса, базисы и фундаментальный бидифференциал. Теорема Вейерштрасса о пробелах гласит (см. [39]):

TeOpema 3.1 (Lückensatz). Для любой точки $p \in X$ на несингулярной алгебраической кривой $X$ рода $g$ существует в точности $g$ различных неотрицательных целых чисел $n_{1}, \ldots, n_{g}$, удовлетворяющих неравенствам

$$
n_{1}=1<n_{2}<\cdots<n_{g}<2 g,
$$

таких, что никакая мероморфная функиия на $X$ не может иметь дивизора полюсов исключительно в р степеней $\left(n_{1}, \ldots, n_{g}\right)$.

Набор целых чисел $\left(n_{1}, \ldots, n_{g}\right)$ называется последовательностью пробелов Вейерштрасса и будет обозначаться $\mathfrak{W}(p)$. Для точки в "общем положении" пробелы есть $1, \ldots, g$. Точка $p \in X$, для которой существует мероморфная функция, имеющая в этой точке полюс порядка меньше $g+1$, называется точкой Вейерштрасса.

Для заданной точки $p_{\infty}$ и локального параметра $\xi(p) 2 g$-мерное пространство $\mathrm{H}_{1}^{*}(X, \mathbb{Z})$, двойственное к группе гомологий $\mathrm{H}_{1}(X, \mathbb{Z})$, может быть отождествлено с пространством мероморфных дифференциалов с полюсами только в $p_{\infty}$ с нулевыми вычетами, а отождествление задается интегрированием по циклам. Базис $\left\{u_{1}, \ldots, u_{g}, \Omega_{n_{1}}, \ldots \Omega_{n_{g}}\right\}$ этого пространства состоит из $g$ элементов $\left\{u_{1}, \ldots, u_{g}\right\}$, задающих базис подпространства $\mathrm{H}^{0}(X, K)$ голоморфных дифференциалов, определенных так, что $u_{j}$ равен нулю с точностью до $\left(n_{j}-1\right)$-го порядка в точке $p_{\infty}$ :

$u_{k}=-\left(\xi(p)^{n_{k}-1}+(\right.$ члены высшего порядка $\left.)\right) \mathrm{d} \xi(p), \quad k=1, \ldots, g, \quad n_{k} \in \mathfrak{W}(p)$,

где члены высшего порядка $\xi(p)^{k}$ состоят только из степеней $k$, для которых $k+1 \notin \mathfrak{W}(p)$.

Остальные базисные элементы $\left\{\Omega_{n_{1}}, \ldots, \Omega_{n_{g}}\right\}$ являются нормированными дифференциалами второго рода, где $\Omega_{k}$ совпадают с определенными в (3.13), (3.14). Они взаимно дуальны при спаривании:

$$
\frac{1}{2 \pi \imath} \iint_{X} u_{j} \wedge \Omega_{n_{k}}=\operatorname{Res}_{p=p_{\infty}}\left(\left(\int_{p_{0}}^{p} u_{j}\right) \Omega_{n_{k}}(p)\right)=\delta_{j k} .
$$


Ненулевые матрицы периодов по $\mathfrak{a}$ - и $\mathfrak{b}$-циклам обозначим

$$
\begin{gathered}
\oint_{\mathfrak{a}_{j}} u_{i}=: \mathfrak{A}_{i j}, \quad \oint_{\mathfrak{b}_{j}} u_{i}=: \mathfrak{B}_{i j}, \\
\frac{1}{2 \pi \imath} \oint_{\mathfrak{b}_{j}} \Omega_{n_{i}}=: C_{i j}=\left(\mathbf{U}_{n_{i}}\right)_{j}, \quad i, j=1, \ldots, g .
\end{gathered}
$$

Столбцы $(g \times g)$-матрицы $\mathbb{C}$ заданы векторами $\mathbf{U}_{n_{i}}$, соответствующими пробелам Вейерштрасса:

$$
\mathbb{C}=\left(\mathbf{U}_{n_{1}}, \ldots, \mathbf{U}_{n_{g}}\right), \quad n_{j} \in \mathfrak{W}\left(p_{\infty}\right) .
$$

Билинейные соотношения Римана, полученные применением теоремы Стокса к 2-формам $u_{j} \wedge u_{k}$ и $u_{j} \wedge \Omega_{k}$ на канонической полигонизации кривой, влекут за собой равенства

$$
\mathfrak{A} \mathfrak{B}^{T}=\mathfrak{B A}^{T}, \quad \mathfrak{A} \mathbb{C}^{T}=\mathbf{1}_{g} .
$$

Связь с базисом $\left\{\omega_{1}, \ldots, \omega_{1}, \Omega_{n_{1}}, \ldots, \Omega_{n_{g}}\right\}$ нормированных дифференциалов, таким образом, описывается формулами

$$
\omega_{i}=\sum_{j=1}^{g} \mathfrak{A}_{i j}^{-1} u_{j}=\sum_{j=1}^{g} C_{j i} u_{j}
$$

а нормированная матрица римановых периодов $\mathbf{T}$ есть

$$
\mathbf{T}:=\mathfrak{A}^{-1} \mathfrak{B}=\mathbb{C}^{T} \mathfrak{B}
$$

Определим векторы

$$
\mathbf{R}_{j}=\mathfrak{A U}_{j}, \quad j=1,2, \ldots
$$

Из (3.20) следует, что дифференциалы $u_{i}$ допускают локальное разложение

$$
u_{i}(p(\xi))=-\sum_{j=n_{i}}^{\infty}\left(\mathbf{R}_{j}\right)_{i} \xi(p)^{j-1} d \xi(p) .
$$

Удобно также ввести нормированный симметричный бидифференциал $\Omega(p, q)$ на $X \times X$ (см. [40], [41], [21]), определенный следующими условиями:

$-\Omega(p, q)$ имеет полюс второго порядка на диагонали $p=q$, где его локальная форма, выраженная в терминах параметров $\xi(p), \xi(q)$, есть

$$
\Omega(p, q)=\left(\frac{1}{(\xi(q)-\xi(p))^{2}}+\sum_{i, j=0}^{\infty} \mu_{i j} \xi(p)^{i} \xi(q)^{j}\right) \mathrm{d} \xi(p) \mathrm{d} \xi(q)
$$

где

$$
\mu_{i j}=\mu_{j i}
$$

- $\Omega(p, q)$ голоморфен вне диагонали по $p$ и по $q$;

- все a-циклы обнуляются:

$$
\oint_{p \in \mathfrak{a}_{j}} \Omega(p, q)=\oint_{q \in \mathfrak{a}_{j}} \Omega(p, q)=0 .
$$


Эти условия однозначно определяют бидифференциал $\Omega(q, p)$, явное представление которого есть

$$
\Omega(p, q)=\mathrm{d}_{p} \mathrm{~d}_{q} \ln \theta(\mathscr{A}(p)-\mathscr{A}(q)+\boldsymbol{\delta})
$$

где $\boldsymbol{\delta}$ - несингулярный нечетный полупериод.

Из определений вытекают также следующие свойства:

- b-циклы заданы нормированными голоморфными дифференциалами $\omega_{j}$, $j=1, \ldots, g$ :

$$
\oint_{p \in \mathfrak{b}_{j}} \Omega(p, q)=2 \pi \imath \omega_{j}(q), \quad \oint_{q \in \mathfrak{b}_{j}} \Omega(p, q)=2 \pi \imath \omega_{j}(p)
$$

- вычеты в $p_{\infty}$ локально определенных бидифференциалов $\xi(p)^{-j} \Omega(p, q)$ и $\xi(q)^{-j} \Omega(p, q)$ заданы нормированным дифференциалом второго рода $\Omega_{j}$ :

$$
\operatorname{Res}_{p=p_{\infty}} \xi(p)^{-j} \Omega(p, q)=-\Omega_{j}(q), \quad \operatorname{Res}_{q=p_{\infty}} \xi(q)^{-j} \Omega(p, q)=-\Omega_{j}(p) ;
$$

- коэффициенты $\mu_{i j}$ в разложении (3.50) связаны с коэффициентами в разложении (3.18) следующим образом:

$$
\mu_{i j}=-Q_{i+1, j+1}
$$

3.3. Модель плоской кривой. Рассмотрим алгебраическую кривую $X$ геометрического рода $g \geqslant 1$, заданную уравнением

$$
X: \quad P(x, y)=0, \quad x, y \in \mathbb{C},
$$

где $P$ - полиномом от $x$ и $y$,

$$
P(x, y)=y^{n}+a_{1}(x) y^{n-1}+\cdots+a_{n}(x),
$$

$a_{k}(x), k=0, \ldots, n,-$ полиномы от $x$ и $n>1$. Предположим, что кривая $X$ имеет точку Вейерштрасса в бесконечности $p_{\infty}$, где координаты $x, y$ локально выражаются как

$$
x=\frac{1}{\xi^{n}}+\cdots, \quad y=\frac{1}{\xi^{s}}+\cdots,
$$

а порядок каждого мономиального члена полинома $P(x, y)$ есть порядок его полюса в $p_{\infty}$. Предположим также, что кривая $X$ может быть представлена в виде

$$
y^{n}-x^{s}+(\text { члены низшего порядка })=0,
$$

где $n, s>2$ - положительные целые числа.

Далее плоские координаты точки $p$ обозначаются $(x(p), y(p))$. При рассмотрении локального разложения вблизи базисной точки $p_{\infty}$ с локальным параметром $\xi(p)$ мы также используем $p(\xi)$ для обозначения точки, и $p(0)=p_{\infty}$. Тогда становится возможным включить главную часть $\Omega$ в явное алгебраическое выражение в терминах коэффициентов кривой $X$. 
ТЕОРема 3.2 [23]. Фундаменталъный бидифференииал может быть выражен в виде

$$
\begin{aligned}
\Omega(p, q) & =\frac{\mathscr{F}(p, q)}{(x(p)-x(q))^{2}} \frac{\mathrm{d} x(p) \mathrm{d} x(q)}{P_{y}(x(p), y(p)) P_{y}(x(q), y(q))}+\sum_{i=1}^{g} \sum_{j=1}^{g} \omega_{i}(p) \gamma_{i j} \omega_{j}(q) \\
& =\frac{\mathscr{F}(p, q)}{(x(p)-x(q))^{2}} \frac{\mathrm{d} x(p) \mathrm{d} x(q)}{P_{y}(x(p), y(p)) P_{y}(x(q), y(q))}+\sum_{i=1}^{g} \sum_{j=1}^{g} u_{i}(p) \varkappa_{i j} u_{j}(q),
\end{aligned}
$$

где $\mathscr{F}(p, q)$ - полиномиальная функиия координат $(x(p), y(p), x(q), y(q))$, являющаяся линейной формой по коэфбициентам полиномов $\left\{a_{0}(x), \ldots, a_{n}(x)\right\}$, определяющих модель плоской кривой $(3.58)$, а симметричные $(g \times g)$-матрицы,$\gamma$ состоят из элементов $\varkappa_{i j}, \gamma_{i j}$, заданных как

$$
\gamma_{i j}=\left(\mathfrak{A}^{T} \varkappa \mathfrak{A}\right)_{i j}=-\oint_{p \in \mathfrak{a}_{i}} \oint_{q \in \mathfrak{a}_{j}} \frac{\mathscr{F}(p, q)}{(x(p)-x(q))^{2}} \frac{\mathrm{d} x(p) \mathrm{d} x(q)}{P_{y}(x(p), y(p)) P_{y}(x(q), y(q))} .
$$

ЗАмЕчАниЕ 3.2. Представление (3.62) фундаментального бидифференциала $\Omega(p, q)$ в гиперэллиптическом случае является классическим и может быть найдено в книгах [23] и [20]. Оно подытожено в [24] и распространено на негиперэллиптические кривые в [42], [43].

ЗАмечАниЕ 3.3. Как уже упоминалось в замечании 1.1, матрица $\varkappa$, возникающая в определении многомерной $\sigma$-функции, определена с точностью до прибавления произвольной симметричной матрицы, скажем, $\chi$. Замена $\varkappa \rightarrow \varkappa+\chi$, тем не менее, не изменяет ни высшую формулу Клейна, ни следующие из этой формулы алгебраические и дифференциальные соотношения между ю-функциями. В последующих примерах будут даны явные выражения для полинома $\mathscr{F}(p, q)$, определяющего фундаментальный бидифференциал $\Omega(p, q)$.

Из (3.62) вытекает следующее утверждение.

СлЕДСТВИЕ 3.1. Коэббиииенты $\mu_{i j}$ в разложении в ряд (3.50) могут быть представлены в виде суммы

$$
\mu_{i j}=-Q_{i+1, j+1}=\mu_{i j}^{\mathrm{alg}}+\mu_{i j}^{\mathrm{trans}}
$$

где $\mu_{i j}^{\text {alg }}$ определяется разложсением

$$
\begin{aligned}
\Omega^{\mathrm{alg}}(p, q) & =\frac{\mathscr{F}(p, q)}{(x(p)-x(q))^{2}} \frac{\mathrm{d} x(p) \mathrm{d} x(q)}{P_{y}(x(p), y(p)) P_{y}(x(q), y(q))} \\
& =\left(\frac{1}{(\xi(q)-\xi(p))^{2}}+\sum_{i, j=0}^{\infty} \mu_{i j}^{\mathrm{alg}} \xi(p)^{i} \xi(q)^{j}\right) \mathrm{d} \xi(q) \mathrm{d} \xi(p),
\end{aligned}
$$

$\xi(p)$ и $\xi(q)$ суть локалъные координаты точек $p$ и $q$, a $\mu_{i j}^{\text {trans }}$ задается формулой

$$
\mu_{i j}^{\text {trans }}=\mathbf{R}_{i+1}^{T} \varkappa \mathbf{R}_{j+1} .
$$


ДокАЗАТЕЛЬСтво. Коэффициенты $\mu_{i j}$ в разложении (3.50) нормированного симметричного бидифференциала $\Omega(p, q)$ (проективная связность) около диагонали $p=q$ могут быть выражены как суммы $\mu_{i j}=\mu_{i j}^{\mathrm{alg}}+\mu_{i j}^{\mathrm{trans}}$. Первый член получен разложением первого из равенств (3.65) как рациональной 2-формы на $X \times X$. Второй член следует из локального разложения по степеням $\xi$ голоморфных дифференциалов $u_{i}$ во втором члене правой части (3.62). Он трансцендентен и задан равенством (3.66). Член $\mu_{i j}^{\text {alg }}$ определяет голоморфную часть разложения рациональной 2-формы, возникающей в первом члене в $(3.61),(3.62)$.

ЗАмЕЧАНИЕ 3.4. Отметим, что двойной интеграл в (3.63) может быть разложен по периодам базисных голоморфных и мероморфных дифференциалов второго рода. Следуя построению Бейкера [23], [44], мы представляем подынтегральное выражение $\Omega^{\mathrm{alg}}(p, q)$ в виде

$$
\begin{aligned}
\Omega^{\operatorname{alg}}(p, q) & =\frac{\mathscr{F}((x, y) ;(z, w))}{(x-z)^{2}} \frac{\mathrm{d} x \mathrm{~d} z}{P_{y}(x, y) P_{z}(z, w)} \\
& =\frac{\partial}{\partial z} \Pi_{(z, w)}^{\left(z^{\prime}, w^{\prime}\right)}(x, y) \mathrm{d} z+\mathbf{u}^{T}(x, y) \mathbf{r}(z, w) .
\end{aligned}
$$

Здесь $\Pi_{(z, w)}^{\left(z^{\prime}, w^{\prime}\right)}(x, y)$ - дифференциал третьего рода с полюсами первого порядка в точках $(z, w)$ и $\left(z^{\prime}, w^{\prime}\right)$ и соответствующими вычетами $\pm 1, \mathbf{u}=$ $\left(u_{1}, \ldots, u_{g}\right)^{T}$ - вектор голоморфных дифференциалов, нормированных как в $(3.40), \mathbf{r}=\left(r_{1}, \ldots, r_{g}\right)^{T}$ - вектор мероморфных дифференциалов с полюсами порядков $n_{1}+1, \ldots, n_{g}+1$ в $p_{\infty}$. (Положение полюса $\left(z^{\prime}, w^{\prime}\right)$ может быть выбрано произвольно и не влияет на построение.) Дифференциалы $\mathbf{r}$ выбраны таким образом, чтобы они удовлетворяли условию симметрии

$$
\Omega^{\mathrm{alg}}(p, q)=\Omega^{\mathrm{alg}}(q, p) .
$$

Явное алгебраическое построение дифференциалов $\mathbf{r}$ описано в [23] и развито далее в [24]. В частности, в случае гиперэллиптической кривой

$$
P(x, y)=y^{2}-\mathscr{P}_{2 g+1}(x),
$$

где $\mathscr{P}_{2 g+1}(x)$ - полином от $x$ степени $2 g+1$, и

$$
\Pi_{(z, w)}^{\left(z^{\prime}, w^{\prime}\right)}(x, y)=\frac{1}{2 y}\left\{\frac{y+w}{x-z}-\frac{y+w^{\prime}}{x-z^{\prime}}\right\} \mathrm{d} x .
$$

Второй член в (3.70) может быть выбран как произвольная конечная точка кривой.

Матрицы $\mathfrak{a}-$ и $\mathfrak{b}$-периодов дифференциалов $\mathbf{r}$, заданные во введении уравнениями (1.21) и (1.20), входят в определение многомерной $\sigma$-функции $(3.72)$ ниже.

3.4. $\sigma$-функции и алгебро-геометрические формулы для $\pi_{\lambda}(w)$. Допустим, что $\mathscr{D}=p_{1}+\cdots+p_{g}-$ положительный неспециальный дивизор степени $g$ и

$$
\mathbf{v}:=\sum_{i=1}^{g} \int_{p_{\infty}}^{q_{i}} \mathbf{u}+\mathfrak{A K}=-\mathfrak{A} \mathbf{e},
$$


где $\mathfrak{A}$ определено в (3.42), $\mathbf{K}$ - вектор римановых констант с базисной точкой в $p_{\infty}$ и $\mathbf{u}=\left(u_{1}, \ldots, u_{g}\right)^{T}$.

Многомерная $\sigma$-функция $\sigma(\mathbf{v})$ определяется формулой

$$
\sigma(\mathbf{v})=C \theta(\mathbf{e}) \exp \left\{\frac{1}{2} \mathbf{v}^{T} \varkappa \mathbf{v}\right\}, \quad \mathbf{e}=-\mathfrak{A}^{-1} \mathbf{v},
$$

где $C$ - константа (зависящая от модулей кривой), явный вид которой нам не потребуется. Это определение естественным образом обобщает на высшие рода $\sigma$-функцию Вейерштрасса из теории эллиптических функций.

Многомерные $\boldsymbol{\zeta}$-функции $\boldsymbol{\zeta}=\left(\zeta_{1}, \ldots, \zeta_{g}\right)$ определяются как

$$
\zeta_{k}(\mathbf{v})=\frac{\partial}{\partial v_{k}} \ln \sigma(\mathbf{v}), \quad k=1, \ldots, g .
$$

Многомерные ю-функции Клейна определяются как вторые логарифмические производные от $\sigma$-функции:

$$
\wp_{i k}(\mathbf{v})=-\frac{\partial^{2}}{\partial v_{i} \partial v_{k}} \ln \sigma(\mathbf{v}), \quad i, k=1, \ldots, g .
$$

Более общим образом, для логарифмических производных высшего порядка мы используем обозначение

$$
\wp_{m_{1}}^{i_{1_{1}, \ldots, i_{1}}, \ldots, \underbrace{i_{k}, \ldots, i_{k}}_{m_{k}}}(\mathbf{v})=-\frac{\partial^{m_{1} i_{1}+\cdots+m_{k} i_{k}}}{\partial v_{i_{1}}^{m_{1}} \cdots \partial v_{i_{k}}^{m_{k}}} \ln \sigma(\mathbf{v}), \quad i_{1}, \ldots, i_{k} \in\{1, \ldots, g\} .
$$

В классической теории следующая теорема дает базовый метод вывода алгебраических и дифференциальных соотношений между многомерными ю-функциями. (См., например, [23] и более современное изложение [24].)

Теорема 3.3 (формула Клейна). Пусть плоская кривая X рода g определена полиномиальным уравнением $P(x, y)=0$. Выберем независимые голоморфные дифференииалы в виде

$$
u_{k}=\frac{\phi_{k}(x, y)}{f_{y}(x, y)} d x, \quad k=1, \ldots, g,
$$

где $\phi_{k}(x, y)$ - полиномь по $x$ и $у$. Пусть $p=(x, y)$ - произвольная точка на $X$ и $\mathscr{D}=p_{1}+p_{2}+\cdots+p_{g}-$ положительный неспециальный дивизор на $X$, $p_{k}=\left(x_{k}, y_{k}\right)$. Пусть v - образ $\mathscr{D}$ при отображении Абеля, сдвинутый как в (3.71). Тогда

$$
\sum_{j, k=1}^{g} \wp_{j k}\left(\int_{p_{0}}^{p} \mathbf{u}-\mathbf{v}\right) \phi_{k}(x, y) \phi_{j}\left(x_{r}, y_{r}\right)=\frac{\mathscr{F}\left(p, p_{r}\right)}{\left(x-x_{r}\right)^{2}}, \quad r=1, \ldots, g
$$

где полином $\mathscr{F}\left(p, p_{r}\right)=\mathscr{F}\left((x, y) ;\left(x_{r}, y_{r}\right)\right)$ определяет фундаментальньй бидифференциал $\Omega\left(p, p_{r}\right)$. 
ЗАмЕчАниЕ 3.5. Эта формула была впервые получена для гиперэллиптических кривых в [40], [41]. Но доказательство, основанное на теореме Римана об обращении в нуль и представлении фундаментального бидифференциала в виде (3.62), может быть легко распространено на общий случай.

Теорема Вейерштрасса-Пуанкаре (см., например, [45]) утверждает, что любые $g+1$ абелевы функции на якобиане алгебраической кривой рода $g$ являются алгебраически зависимыми. В частности, отсюда следует, что в случае гиперэллиптической кривой рода $g$ среди $(g+1) g / 2$ функций $\wp_{i j}$ существует только $g$ алгебраически независимых, поэтому эти функции должны удовлетворять $g(g-1) / 2$ соотношениям. (С использованием формулы Клейна (3.76) в [24] было показано, что эти соотношения являются поверхностями четвертого порядка, представляющими многообразие Куммера, см. пример кривой рода 2 в п. 4.1 ниже.)

Другой набор соотношений, который следует из формулы Клейна, описывает многообразие Якоби кривой $X$ и интегрируемые потоки типа КП, используя $\wp$-функции в качестве координат. В частности, в случае гиперэллиптических кривых все произведения $\wp_{i j k} \wp_{p q r}$ являются кубическими полиномами от $\wp_{i j}$. (См. детали в [24].) Здесь мы покажем, что эти результаты могут также быть получены в рамках теории $\tau$-функций на базе формулы Сато или, что равносильно, из билинейного тождества. Для этого мы представим $\tau$-функцию Сато в терминах многомерной $\sigma$-функции Клейна. Эти методы вывода интегрируемых иерархий типа КП многообразий Якоби и Куммера сравниваются в [46].

ПРЕДЛОЖЕНИЕ 3.1. Нормированная алгебро-геометрическая $\tau$-функиия въражается в терминах многомерной $\sigma$-функиии как

$$
\frac{\tau(\mathbf{e}, \mathbf{t})}{\tau(\mathbf{e}, \mathbf{0})}=\frac{\sigma\left(\sum_{k=1}^{\infty} \mathbf{R}_{k} t_{k}+\mathbf{v}\right)}{\sigma(\mathbf{v})} \exp \left\{\sum_{k=1}^{\infty} \Lambda_{k}(\mathbf{v}) t_{k}\right\} \exp \left\{\frac{1}{2} \sum_{k, l=1}^{\infty} \mu_{k l}^{\mathrm{alg}} t_{k} t_{l}\right\} .
$$

Здесъ $\mathbf{v}=\mathfrak{A} \mathbf{e}-$ сдвинутый абелев образ (3.71) положительного неспециального дивизора $\mathscr{D}$ и $\mu_{i k}^{\text {alg }}$ - коэфбичиенты в разложении алгебраической части бидифберенииала $\Omega(p, q)$ вблизи точки $p_{\infty}$. Коэффициенты $\Lambda_{k}(\mathbf{v})$ заданы равенствами

$$
\Lambda_{k}(\mathbf{v})=\mathbf{R}_{k}^{T} \varkappa \mathbf{v}, \quad k=1,2, \ldots
$$

ДокАЗАТЕльство. Алгебро-геометрическая $\tau$-функция в калибровочно эквивалентном виде (3.37) приводит к соотношению

$$
\frac{\tau(\mathbf{e}, \mathbf{t})}{\tau(\mathbf{e}, \mathbf{0})}=\exp \left\{\frac{1}{2} \sum_{i, j=0}^{\infty} \mu_{i j}^{\mathrm{alg}} t_{i} t_{j}+\frac{1}{2} \sum_{i, j=0}^{\infty} \mu_{i j}^{\mathrm{trans}} t_{i} t_{j}\right\} \frac{\theta\left(\mathbf{e}+\sum_{i=1}^{\infty} \mathbf{U}_{i} t_{i}\right)}{\theta(\mathbf{e})},
$$

где $\lambda_{i}$ заданы в (3.33) и использовано соотношение (3.56).

Действуя иначе, из определения $\sigma$-функции получаем

$$
\begin{aligned}
\sigma\left(\sum_{k=1}^{\infty} \mathbf{R}_{k} t_{k}+\mathbf{v}\right)= & C \theta\left(\sum_{k=1}^{\infty} \mathbf{U}_{k} t_{k}+\mathfrak{A}^{-1} \mathbf{v}\right) \exp \left\{\frac{1}{2} \sum_{k, l=1}^{\infty} \mathbf{R}_{k}^{T} \varkappa \mathbf{R}_{l} t_{k} t_{l}\right\} \\
& \times \exp \left\{\sum_{k=1}^{\infty} \mathbf{R}_{k}^{T} \varkappa \mathbf{v} t_{k}\right\} \exp \left\{\frac{1}{2} \mathbf{v}^{T} \varkappa \mathbf{v}\right\} .
\end{aligned}
$$


Здесь $C$ - константа из определения $\sigma$-функции (3.72). Учитывая (3.66), мы получаем представление (3.77) с коэффициентами $\Lambda_{k}$, заданными в (3.78).

Так как $\tau$-функция определена только с точностью до линейного по $\mathbf{t}$ экспоненциального множителя, мы опускаем линейный член в экспоненте (3.77), с тем чтобы получить более простую формулу

$$
\frac{\tau(\mathbf{e}, \mathbf{t})}{\tau(\mathbf{e}, \mathbf{0})}=\frac{\sigma\left(\sum_{k=1}^{\infty} \mathbf{R}_{k} t_{k}+\mathbf{v}\right)}{\sigma(\mathbf{v})} \exp \left\{\frac{1}{2} \sum_{k, l=1}^{\infty} \mu_{k l}^{\mathrm{alg}} t_{k} t_{l}\right\}, \quad \mathbf{v}=-\mathfrak{A} \mathbf{e}
$$

ЗАмЕчАниЕ 3.6. Похожая формула для алгебро-геометрических $\tau$-функций в терминах $\sigma$-функции была дана А. Накаяшики в [31], где также учитываются члены в экспоненте, линейные по $t_{k}$.

На первый взгляд, использование многомерной $\sigma$-функции вместо $\theta$-функции Римана в выражении для алгебро-геометрической $\tau$-функции кажется тривиальной заменой. Но результатом является то, что квадратичная форма в аргументе экспоненты имеет коэффициенты, выражающиеся алгебраически в терминах коэффициентов кривой. Более того, эти коэффициенты $\mu_{k l}^{\text {alg }}$ являются полиномами от коэффициентов кривой (см. детали в [47]). Как показано ниже, такое представление $\tau$-функции в качестве результата дает решения интегрируемой иерархии КП, выраженные как дифференциальные полиномы от ю-функций с полиномиальными коэффициентами, определенными непосредственно в терминах коэффициентов полиномов $P(x, y)$, задающих кривую.

Согласно предложениям 2.1 и 2.2 , для любой кривой $X$ рода $g$ ассоциированная алгебро-геометрическая $\tau$-функция допускает следующее разложение:

$$
\frac{\tau(\mathbf{e}, \boldsymbol{t})}{\tau(\mathbf{e}, \mathbf{0})}=\sum_{\lambda} \pi_{\lambda}(w) s_{\lambda}(\mathbf{t})
$$

где

$$
\pi_{\lambda}(w)=\left.(-1)^{\sum_{k=1}^{r} b_{k}} \operatorname{det}\left(A_{a_{i} b_{j}}\right)\right|_{1 \leqslant i, j \leqslant r},
$$

сумма берется по всем разбиениям $\lambda$, в обозначениях Фробениуса имеющих вид $\left(a_{1}, \ldots, a_{r} \mid b_{1}, \ldots, b_{r}\right)$. Здесь $A_{i j}, i, j=0, \ldots, \infty,-$ элементы матрицы аффинных координат $A$, представляющей элемент грассманиана $w\left(X, \mathscr{D}, p_{\infty}, \zeta\right) \in$ $\operatorname{Gr}_{\mathscr{H}_{+}}(\mathscr{H})$.

СлеДСтвиЕ 3.2. Элементы $A_{i j}$ могут быть представлены как полиномь от символов Клейна

$$
\zeta_{i}(\mathbf{v}), \wp_{i j}(\mathbf{v}), \ldots, \quad i, j \in\{1, \ldots, g\},
$$

и коэффичиентов полинома $P(x, y)$.

ДокАЗАТЕльство. Величины $\pi_{\lambda}(w)$ в $\tau$-разложении (3.82) можно выразить в терминах отношений $\sigma_{i}(\mathbf{v}) / \sigma(\mathbf{v}), \sigma_{i j}(\mathbf{v}) / \sigma(\mathbf{v})$ и т. д. Но для $i, j, k, \ldots=1, \ldots, g$ 
мы получаем

$$
\begin{gathered}
\frac{\sigma_{i}(\mathbf{v})}{\sigma(\mathbf{v})}=\zeta_{i}(\mathbf{v}), \quad \frac{\sigma_{i j}(\mathbf{v})}{\sigma(\mathbf{v})}=\zeta_{i}(\mathbf{v}) \zeta_{j}(\mathbf{v})-\wp_{i j}(\mathbf{v}) \\
\frac{\sigma_{i j k}(\mathbf{v})}{\sigma(\mathbf{v})}=\zeta_{i}(\mathbf{v})(\mathbf{v}) \wp_{j k}(\mathbf{v})+\zeta_{j}(\mathbf{v})(\mathbf{v}) \wp_{i k}(\mathbf{v})+\zeta_{k}(\mathbf{v})(\mathbf{v}) \wp_{i j}(\mathbf{v}) \\
-\zeta_{i}(\mathbf{v}) \zeta_{j}(\mathbf{v}) \zeta_{k}(\mathbf{v})+\wp_{i j k}(\mathbf{v})
\end{gathered}
$$

Каждому символу $\wp_{k_{1} \ldots k_{g}}$ мы приписываем вес:

$$
\underbrace{\underbrace{}_{1}, \ldots, 1}_{k_{1}}, \underbrace{2, \ldots, 2}_{k_{2}}, \ldots, \underbrace{g, \ldots, g}_{k_{g}} \Leftrightarrow \mathscr{W}_{k_{1} \ldots k_{g}}=\sum_{j=1}^{g} k_{j} n_{j},
$$

где $\left\{n_{i}\right\}_{i=1, \ldots, g}$ - последовательность пробелов Вейерштрасса в бесконечности.

Каждому коэффициенту $a_{k l}$ мономиального члена $a_{k l} x^{k} y^{l}, k<s, l<n$, полинома $P(x, y)$, определяющего кривую (3.57), мы приписываем вес $\widehat{\mathscr{W}}_{k l}$ :

$$
a_{k l} \quad \Leftrightarrow \quad \widehat{\mathscr{W}}_{k l}=n s-(n k+l s) .
$$

Наконец, припишем моному, являющемуся произведением ю-символов с коэффициентами $a_{k, j}$, вес $\mathscr{W}$, который является суммой весов множителей:

$$
a_{i j} \cdots a_{k l} \wp_{i_{1} \ldots i_{g}} \cdots \wp_{k_{1} \ldots k_{g}} \Leftrightarrow \mathscr{W}=\widehat{\mathscr{W}}_{i j}+\cdots+\widehat{\mathscr{W}}_{k l}+\mathscr{W}_{i_{1} \ldots i_{g}}+\cdots+\mathscr{W}_{k_{1} \ldots k_{g}} .
$$

Рассмотрим набор соотношений (типа Джамбелли)

$$
\pi_{\left(a_{1}, \ldots, a_{r} \mid b_{1}, \ldots, b_{r}\right)}=(-1)^{\sum_{i=1}^{r} b_{i}} \operatorname{det}\left(A_{a_{i} b_{j}}\right),
$$

соответствующих разбиению $\lambda=\left(a_{1}, \ldots, a_{r} \mid b_{1}, \ldots, b_{r}\right)$ веса $\mathscr{W}$. Процедура, описанная выше, сводит соотношения (3.90) к соответствующим однородным полиномиальным соотношениям веса $\mathscr{W}$ между ю-функциями $\wp_{i_{1} \ldots i_{k}}$, коэффициенты которых полиномиально зависят от коэффициентов полинома $P(x, y)$, задающего кривую. Такие соотношения описывают иерархии типа КП в терминах ю-координат.

\section{4. Примеры и приложения разложений по функциям Шура}

4.1. $\tau$-функция гиперэллиптической кривой. Пусть $X$ - гиперэллиптическая кривая рода $g$, заданная уравнением

$$
X: \quad P(x, y)=0,
$$

где полином $P(x, y)$ имеет вид

$$
P(x, y)=y^{2}-4 x^{2 g+1}-\cdots-\alpha_{0}=y^{2}-4 \prod_{j=1}^{2 g+1}\left(x-a_{j}\right) .
$$


Как и выше, $p=(x, y)$ будет обозначать произвольную точку кривой $X$ и $p_{\infty}=$ $(\infty, \infty)$. Выберем канонический базис циклов $\left(\mathfrak{a}_{1}, \ldots, \mathfrak{a}_{g} ; \mathfrak{b}_{1}, \ldots, \mathfrak{b}_{g}\right) \in H_{1}(X, \mathbb{Z})$ и зафиксируем базисные голоморфные дифференциалы $\mathbf{u}=\left(u_{1}, \ldots, u_{g}\right)^{T}$ как

$$
u_{i}(p)=\frac{x^{i-1} \mathrm{~d} x}{y}, \quad i=1, \ldots, g .
$$

Как и выше, обозначим матрицы периодов через $\mathfrak{A}, \mathfrak{B}$ и положим $\mathbf{T}=\mathfrak{A}^{-1} \mathfrak{B}$.

Пусть $\mathscr{D}=q_{1}+\cdots+q_{g}-$ неспециальный дивизор степени $g$, и пусть $\mathbf{v}-$ сдвинутое отображение Абеля, заданное в (3.71). В этом случае вектор римановых констант $\mathbf{K}$ может быть задан как образ отображения Абеля дивизора $\mathscr{D}=\left(p_{1}, \ldots, p_{g}\right)$, где $p_{k}=\left(a_{k}, 0\right)$ - точки ветвления, абелевы образы которых являются нечетными полупериодами [39; разд. VII.1.2]:

$$
\mathbf{K}=-\sum_{k=1}^{g} \int_{p_{\infty}}^{p_{k}} \boldsymbol{\omega}=-\mathfrak{A}^{-1} \sum_{k=1}^{g} \int_{p_{\infty}}^{p_{k}} \mathbf{u} .
$$

Поэтому мы можем записать

$$
\mathbf{v}=\sum_{k=1}^{g} \int_{\left(a_{k}, 0\right)}^{q_{k}} \mathbf{u}
$$

В классической теории (см., например, [23], [44]) было показано, что квадратичный бидифференциал $\Omega(p, q)$ может быть выбран в виде

$$
\Omega(p, q)=\frac{F(x, z)+2 y w}{4(x-z)^{2} y w} \mathrm{~d} x \mathrm{~d} z+2 \mathbf{v}^{T}(p) \varkappa \mathbf{v}(q),
$$

где полином $F(x, z)$ является 2-полярой Клейна:

$$
F(x, z)=\sum_{m=0}^{g} x^{m} z^{m}\left(2 \alpha_{2 m}+(x+z) \alpha_{2 m+1}\right),
$$

и симметричная $(g \times g)$-матрица $\varkappa$ задана формулой $\varkappa=\mathfrak{A}^{-1} \mathfrak{S}$, где $\mathfrak{S}$ - матрица a-периодов мероморфных дифференциалов

$$
r_{j}=\sum_{k=j}^{2 g+1-j}(k+1-j) \alpha_{k+1+j} \frac{x^{k} \mathrm{~d} x}{4 y}, \quad j=1, \ldots, g .
$$

ТЕОРемА 4.1 (формула Клейна для гиперэллиптической кривой). Пусть плоская кривая $X$ задана полиномиалъным уравнением (4.2). Пусть $p=(x, y)-$ произвольная точка кривой $X$ и $\mathscr{D}=p_{1}+\cdots+p_{g}-$ неспециальный дивизор на $X, p_{k}=\left(x_{k}, y_{k}\right)$. Пусть $\mathbf{v}$ - сдвинутое отображение Абеля дивизора $\mathscr{D}$, заданное в (4.5). Тогда

$$
\sum_{i, k=1}^{g} \wp_{i k}\left(\mathscr{A}(p)-\mathscr{A}\left(p_{\infty}\right)+\mathbf{v}\right) x^{k-1} x_{r}^{i-1}=\frac{F\left(x, x_{r}\right)-2 y y_{r}}{4\left(x-x_{r}\right)^{2}}, \quad r=1, \ldots, g
$$


где полином

$$
\mathscr{F}\left(p, p_{r}\right)=\mathscr{F}\left((x, y) ;\left(x_{r}, y_{r}\right)\right)=F\left(x, x_{r}\right)-2 y y_{r}
$$

определяет фундаментальный бидифференииал $\Omega\left(p, p_{r}\right)$.

ЗАмечАниЕ 4.1. Дифференциальные соотношения на $\wp_{i j}$ описывают все возможные интегрируемые уравнения, ассоциированные с заданной кривой. Более того, можно показать, что для произвольного рода $g$ любая четная производная $\wp_{i_{1} \ldots i_{2 k}}, k>1$, может быть записана как полином от $\wp_{i k}$ с коэффициентами, выраженными в терминах инвариантов кривой [24]. Полный набор дифференциальных соотношений в частных случаях $g=2$ и $g=3$ можно найти в [48], [20], а недавно эти соотношения в ковариантной форме были получены в работе [49].

Теперь ограничимся случаем кривой рода два, уравнение которой может быть записано в виде

$$
\begin{aligned}
y^{2} & =4 x^{5}+\alpha_{4} x^{4}+\alpha_{3} x^{3}+\alpha_{2} x^{2}+\alpha_{1} x+\alpha_{0} \\
& =4\left(x-a_{1}\right)\left(x-a_{2}\right)\left(x-a_{3}\right)\left(x-a_{4}\right)\left(x-a_{5}\right), \quad a_{i} \neq a_{j} .
\end{aligned}
$$

Голоморфные дифференциалы $\mathbf{u}=\left(u_{1}, u_{2}\right)^{T}$ связаны с $\mathbf{v}=\left(v_{1}, v_{2}\right)^{T}$, где

$$
v_{1}=\frac{x \mathrm{~d} x}{y}, \quad v_{2}=\frac{\mathrm{d} x}{y},
$$

преобразованием

$$
\left(\begin{array}{cc}
1 & \alpha_{4} / 8 \\
0 & 1
\end{array}\right)
$$

Согласно определению $(3.65)$, первые несколько коэффициентов $\mu_{i j}^{\mathrm{alg}}$ имеют следующий вид:

$$
\begin{aligned}
& \mu_{i j}^{\mathrm{alg}}=0, \quad \text { если } i \text { или } j \text { или они оба четные, } \\
& \mu_{11}^{\mathrm{alg}}=-\frac{1}{16} \alpha_{4}, \\
& \mu_{13}^{\mathrm{alg}}=\mu_{31}=-\frac{1}{16} \alpha_{3}+\frac{3}{256} \alpha_{4}^{2}, \\
& \mu_{15}^{\mathrm{alg}}=\mu_{51}=-\frac{1}{16} \alpha_{2}+\frac{3}{128} \alpha_{3} \alpha_{4}-\frac{5}{2048} \alpha_{4}^{3}, \\
& \mu_{33}^{\mathrm{alg}}=-\frac{3}{16} \alpha_{2}+\frac{1}{32} \alpha_{3} \alpha_{4}-\frac{3}{1024} \alpha_{4}^{3},
\end{aligned}
$$

Введем набор векторов вычетов

$$
\mathbf{R}_{2 k}=0, \quad k=1,2, \ldots,
$$

И

$$
\mathbf{R}_{1}=\left(\begin{array}{l}
1 \\
0
\end{array}\right), \quad \mathbf{R}_{3}=\left(\begin{array}{c}
-\alpha_{4} / 8 \\
1
\end{array}\right), \quad \mathbf{R}_{5}=\left(\begin{array}{c}
-\alpha_{3} / 8+3 \alpha_{4}^{2} / 128 \\
-\alpha_{4} / 8
\end{array}\right), \quad \ldots
$$


Из формул (2.48) и (2.60) следует, что первые несколько компонент аффинной матрицы имеют вид

$$
\begin{aligned}
& A_{00}(\mathbf{v})=\zeta_{1}, \\
& A_{01}(\mathbf{v})=\frac{1}{2} \zeta_{1}^{2}-\frac{1}{2} \wp_{11}-\frac{1}{16} \alpha_{4}, \\
& A_{02}(\mathbf{v})=\frac{1}{6} \zeta_{1}^{3}+\frac{1}{3} \zeta_{2}-\left(\frac{1}{2} \wp_{11}+\frac{5}{48} \alpha_{4}\right) \zeta_{1}-\frac{1}{6} \wp_{111}, \\
& A_{03}(\mathbf{v})=\frac{1}{24} \zeta^{4}+\frac{1}{3} \zeta_{1} \zeta_{2}-\left(\frac{7}{96} \alpha_{4}+\frac{1}{4} \wp_{11}\right)-\frac{1}{6} \wp_{111} \zeta_{1} \\
& -\frac{1}{24} \wp_{1111}-\frac{1}{3} \wp_{12}+\frac{1}{8} \wp_{11}^{2}+\frac{7}{96} \alpha_{4} \wp_{11}-\frac{1}{24} \alpha_{3}+\frac{5}{512} \alpha_{4}^{2}, \\
& A_{10}(\mathbf{v})=A_{01}(\mathbf{v}), \\
& A_{11}(\mathbf{v})=\frac{1}{3} \zeta_{1}^{3}-\frac{1}{3} \zeta_{2}-\left(\wp_{11}+\frac{1}{12} \alpha_{4}\right) \zeta_{1}-\frac{1}{3} \wp_{111}, \\
& A_{12}(\mathbf{v})=\frac{1}{8} \zeta_{1}^{4}-\frac{1}{2} \zeta_{1} \wp_{111}+\frac{3}{8} \wp_{11}^{2}-\frac{1}{8} \wp_{1111}-\left(\frac{3}{4} \wp_{11}+\frac{3}{32} \alpha_{4}\right) \zeta_{1}^{2} \\
& +\frac{3}{32} \alpha_{4} \wp_{11}+\frac{3}{512} \alpha_{4}^{2} \\
& A_{13}(\mathbf{v})=\frac{1}{30} \zeta_{1}^{5}+\frac{1}{6} \zeta_{1}^{2} \zeta_{2}-\frac{1}{3} \wp_{111} \zeta_{1}^{2} \\
& -\left(\frac{1}{3} \wp_{11}+\frac{1}{16} \alpha_{4}\right) \zeta_{1}^{3}-\left(\frac{1}{6} \wp_{11}+\frac{1}{48} \alpha_{4}\right) \zeta_{2} \\
& +\left(-\frac{1}{6} \wp_{1111}-\frac{1}{3} \wp_{12}+\frac{1}{2} \wp_{11}^{2}+\frac{3}{16} \alpha_{4} \wp_{11}-\frac{1}{24} \alpha_{3}+\frac{7}{384} \alpha_{4}^{2}\right) \zeta_{1} \\
& +\frac{1}{3} \wp_{11} \wp_{111}-\frac{1}{6} \wp_{1112}-\frac{1}{30} \wp_{11111}+\frac{1}{16} \alpha_{4} \wp_{111},
\end{aligned}
$$

Все символы Клейна, $\zeta_{k}, \wp_{k l}$ и т. д., в этих формулах вычисляются в точке v.

ПРЕДЛОЖЕНИЕ 4.1. Плюккеровы соотношения, записанные для разбиения $\lambda=(2,2)$ веса 4 и разбиений $\lambda=(3,2), \lambda=(2,2,1)$ веса 5 , эквивалентны уравнению

$$
\wp_{1111}(\mathbf{v})=6 \wp_{11}^{2}(\mathbf{v})+4 \wp_{12}(\mathbf{v})+\alpha_{4} \wp_{11}(\mathbf{v})+\frac{1}{2} \alpha_{3} .
$$

ДокАЗАТЕльство. Первое нетривиальное плюккерово соотношение для разбиения $\lambda=(2,2)$,

$$
\pi_{(1,0 \mid 1,0)}=\left|\begin{array}{ll}
A_{11} & A_{10} \\
A_{01} & A_{00}
\end{array}\right|,
$$


записывается в развернутом виде как

$$
\begin{gathered}
\left.\tau(\mathbf{0}, \mathbf{v})\left[\frac{1}{12} \frac{\partial^{4}}{\partial t_{1}^{4}}+\frac{1}{4} \frac{\partial^{2}}{\partial t_{2}^{2}}-\frac{1}{3} \frac{\partial^{2}}{\partial t_{1} \partial t_{3}}\right] \tau(\mathbf{t}, \mathbf{v})\right|_{\mathbf{t}=\mathbf{0}} \\
=\left|\begin{array}{ll}
{\left.\left[\frac{1}{3} \frac{\partial^{3}}{\partial t_{1}^{3}}-\frac{1}{3} \frac{\partial}{\partial t_{3}}\right] \tau(\mathbf{t}, \mathbf{v})\right|_{\mathbf{t}=\mathbf{0}}} & {\left.\left[\frac{1}{2} \frac{\partial}{\partial t_{2}}+\frac{\partial^{2}}{\partial t_{1}^{2}}\right] \tau(\mathbf{t}, \mathbf{v})\right|_{\mathbf{t}=\mathbf{0}}} \\
{\left.\left[-\frac{1}{2} \frac{\partial}{\partial t_{2}}+\frac{1}{2} \frac{\partial^{2}}{\partial t_{1}^{2}}\right] \tau(\mathbf{t}, \mathbf{v})\right|_{\mathbf{t}=\mathbf{0}}} & \left.\frac{\partial}{\partial t_{1}} \tau(\mathbf{t}, \mathbf{v})\right|_{\mathbf{t}=\mathbf{0}}
\end{array}\right|
\end{gathered}
$$

Подставляя в это соотношение выражение (3.81) для $\tau(\mathbf{t}, \mathbf{e})$ и выражения (4.14) для $\mu_{i j}$ и вычисляя производные по направлению, получаем уравнение (4.17). Выражения для коэффициентов диаграмм-крюков, входящие в детерминант, заданы выше и

$$
\begin{aligned}
\pi_{(1,0 \mid 1,0)}=- & \frac{1}{12} \wp_{1111}+\frac{1}{12} \zeta_{1}^{4}-\left(\frac{1}{2} \wp_{11}+\frac{1}{48} \alpha_{4}\right) \zeta_{1}^{2}-\frac{1}{3} \zeta_{2} \zeta_{1}-\frac{1}{3} \wp_{111} \zeta_{1} \\
& +\frac{1}{3} \wp_{12}+\frac{1}{4} \wp_{11}^{2}+\frac{1}{48} \alpha_{4} \wp_{11}-\frac{1}{256} \alpha_{4}^{2}+\frac{1}{24} \alpha_{3}
\end{aligned}
$$

Разбиения $\lambda=(3,2)$ и $\lambda=(2,2,1)$ веса 5 дают плюккеровы соотношения, из которых следует действие выражения $\zeta_{1}(\mathbf{v})+\partial / \partial v_{1}$ на уравнение, приведенное выше. Все это приводит к единственному уравнению:

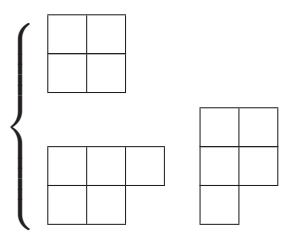

$$
\Leftrightarrow \quad \wp_{1111}(\mathbf{v})=6 \wp_{11}^{2}(\mathbf{v})+4 \wp_{12}(\mathbf{v})+\alpha_{4} \wp_{11}(\mathbf{v})+\frac{1}{2} \alpha_{3}
$$

Аналогичные соображения, касающиеся разбиений весов 6 и 7, ведут к соответствию

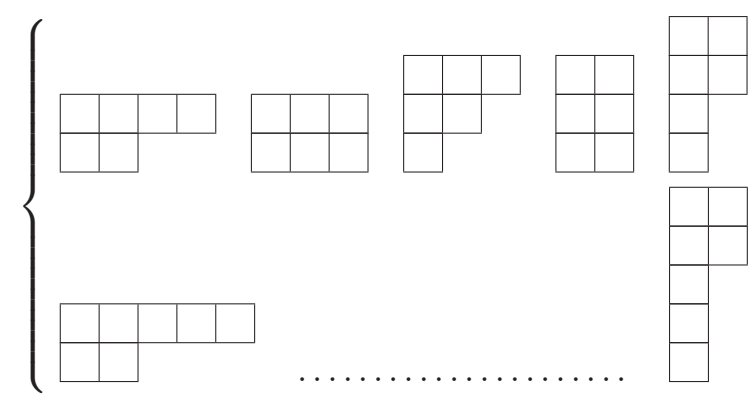

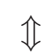

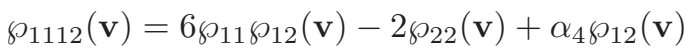

$$
\begin{aligned}
& \wp_{111}^{2}(\mathbf{v})=4 \wp_{11}^{3}(\mathbf{v})+\wp_{22}(\mathbf{v})+4 \wp_{12}(\mathbf{v}) \wp_{11}(\mathbf{v})+\alpha_{4} \wp_{11}^{2}(\mathbf{v})+\alpha_{3} \wp_{11}(\mathbf{v})
\end{aligned}
$$

Используя определение веса $\mathscr{W}$ для функций, возникающих в (4.21) и (4.22), мы видим, что все эти уравнения однородны. 
Описанный здесь процесс может быть продолжен. Кажется разумным предположение, что весь набор дифференциальных соотношений между символами Клейна с мультииндексами может быть сопоставлен с диаграммами Юнга для разбиений $\lambda=\left(2,2, i_{1}, \ldots, i_{n}\right)$ таким образом, что разбиения веса $2 k$ и $2 k+1$ соответствуют набору уравнений веса $\mathscr{W}=2 k$.

Чтобы завершить перечисление основных уравнений, описывающих абелевы функции в терминах плюккеровых соотношений, заметим, что уравнение поверхности Куммера выводится из плюккерова соотношения, соответствующего диаграмме для разбиения $\lambda=(4,4,4,4)$ веса 16 :

$$
\pi_{(3,2,1,0 \mid 3,2,1,0)}=\left|\begin{array}{cccc}
A_{33} & A_{32} & A_{31} & A_{30} \\
A_{23} & A_{22} & A_{21} & A_{20} \\
A_{13} & A_{12} & A_{11} & A_{10} \\
A_{03} & A_{02} & A_{01} & A_{00}
\end{array}\right| .
$$

Уравнение

$$
\pi_{(3,2,1,0 \mid 3,2,1,0)}=0
$$

может быть записано в виде

$$
\left|\begin{array}{cccc}
\alpha_{0} & \alpha_{1} / 2 & -2 \wp_{22} & -2 \wp_{12} \\
\alpha_{1} / 2 & \alpha_{2}+4 \wp_{22} & \alpha_{3} / 2+2 \wp_{12} & -2 \wp_{11} \\
-2 \wp_{22} & \alpha_{3} / 2+2 \wp_{12} & \alpha_{4}+4 \wp_{11} & 2 \\
-2 \wp_{12} & -2 \wp_{11} & 2 & 0
\end{array}\right|=0 .
$$

Таким образом получается замечательная поверхность четвертого порядка, а именно поверхность $\operatorname{Kуммера,~} \operatorname{Kum}(X)$, определенная как поверхность в $\mathbb{C}^{3}$ с координатами $x=\wp_{11}, y=\wp_{12}, z=\wp_{22}$. Она является факторпространством многообразия Якоби: $\operatorname{Kum}(X)=\operatorname{Jac}(X) /(\mathbf{u} \rightarrow-\mathbf{u})$.

Отсюда мы заключаем, что

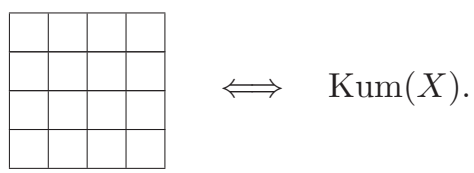

ЗАмечАниЕ 4.2. Уравнение на поверхность Куммера в этом виде было получено Бейкером [20], а обобщение на высшие рода дано в [24]. Заметим также, что уравнения, записанные для всех $(2 \times 2)$-миноров матрицы $\left(A_{i j}\right)_{i, j=0, \ldots, 3}$ B $(4.23)$,

$$
\pi_{(i, k \mid j, l)}=\left|\begin{array}{ll}
A_{i j} & A_{i l} \\
A_{k j} & A_{k l}
\end{array}\right|, \quad i \geqslant j, \quad k \geqslant l, \quad i, j, k, l \in\{0,1,2,3\},
$$

задают полный набор алгебраических уравнений, описывающих многообразие Якоби $\operatorname{Jac}(X)$ как алгебраическое многообразие, а также потоки типа КдФ на $\operatorname{Jac}(X)$. Таким образом, матричное представление многообразий Якоби и Куммера, найденное Бейкером [20] в роде 2 и обобщенное на высшие рода в [24], может быть реализовано в плюккеровых координатах.

Приведенные выше рассуждения приводят к следующему результату. 
Теорема 4.2. Каждъй вектор-столбеи $\mathbf{A}_{k}(\mathbf{v}), k=0,1, \ldots$, в матрице афбфинных координат элемента грассманиана, чъи компоненты $A_{k l}, l=1,2, \ldots$, соответствуют разбиениям-крюкам $\left(k+1,1^{l}\right)$, есть полином от конечного набора символов Клейна $\zeta_{i}(\mathbf{v}), \wp_{i j}(\mathbf{v}), \wp_{i j k}(\mathbf{v})$.

4.2. $\tau$-функция тригональной кривой. В этом пункте мы демонстрируем, как вышеизложенные результаты возникают в случае тригональной кривой. Теория $\sigma$-функций тригональных абелевых функций была развита в [43]. Различные результаты в этой области были получены в [27], [42], [50]-[53]. Чтобы выделить основную идею и избежать громоздких формул, мы ограничимся первым нетривиальным случаем циклического семейства тригональных кривых $X$ рода 3 , определенных уравнением

$$
P(x, y)=y^{3}-\left(x^{4}+\beta_{3} x^{3}+\beta_{6} x^{2}+\beta_{9} x+\beta_{12}\right)=0,
$$

и зафиксируем канонический базис циклов $\left(\mathfrak{a}_{1}, \mathfrak{a}_{2}, \mathfrak{a}_{3} ; \mathfrak{b}_{1}, \mathfrak{b}_{2}, \mathfrak{b}_{3}\right) \in H_{1}(X, \mathbb{Z})$ на $X$.

Явное вычисление канонических голоморфных дифференциалов, а также мероморфных дифференциалов, им сопряженных, дано в [50]. В частности, для $p=(x, y)$ имеем

$$
\begin{gathered}
u_{1}(p)=\frac{\mathrm{d} x}{3 y}, \quad u_{2}(p)=\frac{x \mathrm{~d} x}{3 y^{2}}, \quad u_{3}(p)=\frac{\mathrm{d} x}{3 y^{2}}, \\
r_{1}(p)=\frac{x^{2} \mathrm{~d} x}{3 y^{2}}, \quad r_{2}(p)=-\frac{2 x \mathrm{~d} x}{3 y}, \quad r_{3}(p)=-\frac{\left(5 x^{2}+3 \beta_{3} x+\beta_{6}\right) \mathrm{d} x}{3 y} .
\end{gathered}
$$

Обозначим, как и выше, матрицы периодов через $\mathfrak{A}, \mathfrak{B}$ и положим $\mathbf{T}=\mathfrak{A}^{-1} \mathfrak{B}$, $\varkappa=\mathfrak{A}^{-1} \mathfrak{S}$.

Пусть $\mathscr{D}=p_{1}+p_{2}+p_{3}-$ неспециальный дивизор степени 3 и

$$
\mathbf{v}=\sum_{i=1}^{3} \int_{p_{\infty}}^{p_{i}} \mathbf{u}+\mathfrak{A} \mathbf{K},
$$

где $\mathbf{K}$ - вектор римановых констант с базисной точкой в $p_{\infty}$.

Полином $\mathscr{F}((x, y) ;(z, w))$, возникающий в фундаментальном бидифференциале $\Omega(p, q)$, задается формулой

$$
\mathscr{F}((x, y),(z, w))=3 w^{2} y^{2}+w T(x, z)+y T(z, x),
$$

где

$$
T(x, z)=3 \beta_{12}+(z+2 x) \beta_{9}+x(x+2 z) \beta_{6}+3 \beta_{3} x^{2} z+x^{2} z^{2}+2 x^{3} z .
$$

Разложение около $p_{\infty}$ дает следующие выражения для $\mu_{i j}^{\mathrm{alg}}$ :

$$
\begin{aligned}
\mu_{00}^{\mathrm{alg}} & =0, \\
\mu_{01}^{\mathrm{alg}} & =\mu_{10}^{\mathrm{alg}}=-\frac{2}{3} \beta_{3}, \\
\mu_{04}^{\mathrm{alg}} & =\mu_{40}^{\mathrm{alg}}=-\frac{2}{3} \beta_{6}+\frac{5}{9} \beta_{3}^{2}, \\
\mu_{13}^{\mathrm{alg}} & =\mu_{31}^{\mathrm{alg}}=-\frac{2}{3} \beta_{6}+\frac{4}{9} \beta_{3}^{2}, \\
\mu_{22}^{\mathrm{alg}} & =0,
\end{aligned}
$$


ЗАмЕчАнИЕ 4.3. $\mu_{i j}^{\text {alg }}=0$ для всех $i, j$ кроме тех, для которых $(i+j)+2 \equiv 0$ $\bmod 3$. Это является следствием циклической симметрии кривой.

В этом случае формула Клейна гласит

$$
\sum_{i, k=1}^{3} \wp\left(\int_{p_{\infty}}^{p} \mathbf{u}-\mathbf{v}\right) \phi_{i}(x, y) \phi_{k}\left(x_{r}, y_{r}\right)=\frac{\mathscr{F}\left(p, p_{r}\right)}{\left(x-x_{r}\right)^{2}}, \quad r=1,2,3,
$$

где $p=(x, y), p_{k}=\left(x_{k}, y_{k}\right)$, а также $\phi_{1}(x, y)=y, \phi_{2}(x, y)=x, \phi_{3}(x, y)=1$.

Разложим это соотношение в окрестности $p_{\infty}$, где локальная координата $x=$ $1 / \xi^{3}$ определена. Приравнивая главные части в полюсах, мы получаем набор уравнений на переменные $x_{x}, y_{k}$ и ю-символы. Теперь мы покажем, что эти соотношения могут быть получены как следствия плюккеровых соотношений с помощью формулы типа Джамбелли.

Первая диаграмма Юнга, приводящая к нетривиальному плюккеровому соотношению, как в случае гиперэллиптики рода 2, соответствует разбиению $\lambda=(2,2)$. В рассматриваемом случае после упрощения мы получаем уравнение

$$
\wp_{1111}=6 \wp_{11}^{2}-3 \wp_{22} .
$$

Дифференцирование по координате $v_{1}$ дает уравнение Буссинеска.

Дифференцирование уравнения (4.32) основывается на формуле (4.18) для тригональной кривой (4.27). А именно, мы имеем

$$
\pi_{(1,0 \mid 1,0)}=\frac{1}{4} \wp_{22}+\frac{1}{4} \zeta_{2}^{2}-\frac{1}{12} \wp_{1111}-\frac{1}{3} \wp_{111} \zeta_{1}+\frac{1}{4} \wp_{11}^{2}-\frac{1}{2} \wp_{11} \zeta_{1}^{2}+\frac{1}{12} \zeta_{1}^{4}
$$

и

$$
\begin{aligned}
& A_{00}(\mathbf{v})=\zeta_{1}(\mathbf{v}), \\
& A_{01}(\mathbf{v})=-\frac{1}{2} \wp_{11}(\mathbf{v})+\frac{1}{2} \zeta_{1}^{2}(\mathbf{v})-\frac{1}{2} \zeta_{2}(\mathbf{v}), \\
& A_{10}(\mathbf{v})=\frac{1}{2} \zeta_{2}(\mathbf{v})-\frac{1}{2} \wp_{11}(\mathbf{v})+\frac{1}{2} \zeta_{1}^{2}(\mathbf{v}), \\
& A_{11}(\mathbf{v})=-\frac{1}{3} \wp_{111}(\mathbf{v})-\wp_{11}(\mathbf{v}) \zeta_{1}(\mathbf{v})+\frac{1}{3} \zeta_{1}^{3}(\mathbf{v}) .
\end{aligned}
$$

В тригональном случае, в отличие от случая рода 2 , уже нет симметрии относительно диагонали диаграммы Юнга, но мы можем ограничиться уравнениями четной степени, взяв симметрические комбинации двух диаграмм, связанных транспонированием. В случае веса 5 мы имеем симметрическую комбинацию

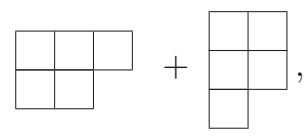

которая дает тригональное дифференциальное уравнение в частных производных веса 5

$$
\wp_{1112}=6 \wp_{11} \wp_{12}+3 \beta_{3} \wp_{11} .
$$

Для веса 6 мы имеем три набора диаграмм
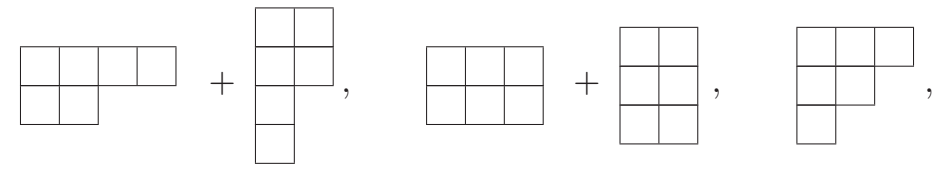
которые приводят к переопределенному набору уравнений с единственным решением

$$
\begin{aligned}
\wp_{111}^{2} & =4 \wp_{11}^{3}+\wp_{12}^{2}+4 \wp_{13}-4 \wp_{11} \wp_{22}, \\
\wp_{1122} & =4 \wp_{13}+4 \wp_{12}^{2}+2 \wp_{11} \wp_{22}+3 \beta_{3} \wp_{12}+2 \beta_{6} .
\end{aligned}
$$

Продолжая в том же духе, можно получить полный набор уравнений для мультииндексных функций ६ циклической тригональной кривой, приведенный в работе [50].

Эта работа выполнена при поддержке European Science Foundation Programme MISGAM (Methods of Integrable System, Geometry and Applied Mathematics). Авторы благодарны HWK, Hanse-Wissenschaftskolleg (Institute of Advanced Study), Delmenhorst за грант, позволивший им работать вместе в мае 2010 г. в HWK над завершением итоговой версии данной работы. Работа первого автора была частично поддержана Natural Science and Engineering Research Council, Canada (NSERC) и Fonds Québécois de la recherche sur la nature et les technologies (FQRNT). Авторы также благодарны Luc Haine за полезные обсуждения и за привлечение внимания к неопубликованному манускрипту Дж. Фея [29].

\section{Список литературы}

[1] С. П. Новиков, "Периодическая задача для уравнения Кортевега-де Фриза. I", Функи. анализ и его прил., 8:3 (1974), 54-66; англ. пер.: S.P. Novikov, "The periodic problem for the Korteweg-de Vries equation", Funct. Anal. Appl., 8:3 (1974), $236-246$.

[2] Б.А. Дубровин, С. П. Новиков, "Периодический и условно периодический аналоги многосолитонных решений уравнения Кортевега-де Фриза", ЖЭТФ, 67:6 (1974), 2131-2144; англ. пер.: В. A. Dubrovin, S.P. Novikov, "Periodic and conditionally periodic analogs of the many-soliton solutions of the Korteweg-de Vries equation", Soviet Physics JETP, 40:6 (1974), 1058-1063.

[3] Б.А. Дубровин, "Обратная задача теории рассеяния для периодических конечнозонных потенциалов", Функц. анализ и его прил., 9:1 (1975), 65-66; B. A. Dubrovin, "Inverse problem for periodic finite-zoned potentials in the theory of scattering", Funct. Anal. Appl., 9:1 (1975), 61-62.

[4] А.Р. Итс, В.Б. Матвеев, "Операторы Шредингера с конечнозонным спектром и $N$-солитонные решения уравнения Кортевега-де Фриса”, ТМФ, 23:1 (1975), 51-68; англ. пер.: A. R. Its, V. B. Matveev, "Schrödinger operators with finite-gap spectrum and $N$-soliton solutions of the Korteweg-de Vries equation", Theoret. Math. Phys., 23:1 (1975), 343-355.

[5] А. Р. Итс, В. Б. Матвеев, "On a class of solutions of the Korteweg-de Vries equation", Problems in mathematical physics, Изд-во Ленингр. ун-та, Л., 1976, 70-92.

[6] В. А. Марченко, "Периодические решения уравнения Кортевега-де Фриза", Докл. AH CCCP, 217 (1974), 276-279; англ. пер.: V. A. Marchenko, "Periodic solutions of the KdV equation", Soviet Math. Dokl., 15 (1974), 1052-1056.

[7] P. D. Lax, "Periodic solutions of the KdV equation", Comm. Pure Appl. Math., 28:1 (1975), 141-188.

[8] H. P. McKean, P. Moerbeke, "The spectrum of Hill's equation", Invent. Math., 30:3 (1975), 217-274.

[9] E. Date, S. Tanaka, "Periodic multi-soliton solutions of Korteweg-de Vries equation and Toda lattice", Progr. Theoret. Phys. Suppl., 1976, № 59, 107-125. 
[10] Б.А. Дубровин, В.Б. Матвеев, С.П. Новиков, "Нелинейные уравнения типа Кортевега-де Фриза, конечнозонные линейные операторы и абелевы многообразия", УМН, 31:1 (1976), 55-136; англ. пер.: B. A. Dubrovin, V. B. Matveev, S.P. Novikov, "Non-linear equations of Korteweg-de Vries type, finite-zone linear operators, and Abelian varieties", Russian Math. Surveys, 31:1 (1976), 59-146.

[11] И. М. Кричевер, "Методы алгебраической геометрии в теории нелинейных уравнений", УМH, 32:6 (1977), 183-208; англ. пер.: I. M. Krichever, "Methods of algebraic geometry in the theory of non-linear equations", Russian Math. Surveys, 32:6 (1977), 185-213.

[12] Б. А. Дубровин, “Тэта-функции и нелинейные уравнения”, УМН, 36:2 (1981), 11-80; англ. пер.: В. A. Dubrovin, "Theta functions and non-linear equations", Russian Math. Surveys, 36:2 (1981), 11-92.

[13] M. Sato, Y. Mori, "On Hirota's bilinear equations. I", RIMS Kôkyûroku, 388 (1980), 183.

[14] M. Sato, Y. Sato, "On Hirota's bilinear equations. II", RIMS Kôkyûroku, 414 (1981), 181.

[15] M. Sato, Y. Sato, "Soliton equations as dynamical systems on infinite-dimensional Grassmann manifold", Nonlinear partial differential equations in applied science (Tokyo, 1982), North-Holland Math. Stud., 81, North-Holland, Amsterdam, 1983, 259-271.

[16] E. Date, M. Jimbo, M. Kashiwara, T. Miwa, "Transformation groups for soliton equations", Nonlinear integrable systems - classical theory and quantum theory (Kyoto, 1981), eds. M. Jimbo and T. Miwa, World Sci. Publ., Singapore, 1983, 39-119.

[17] G. Segal, G. Wilson, "Loop groups and equations of KdV type", Inst. Hautes Études Sci. Publ. Math., 1985, №61, 5-65.

[18] I. G. Macdonald, Symmetric functions and Hall polynomials, Oxford Math. Monogr., The Clarendon Press, New York, 1995, ISBN: 0-19-853489-2, x+475 pp.

[19] B. E. Sagan, The symmetric group. Representations, combinatorial algorithms, and symmetric functions, 2nd ed., Grad. Texts in Math., 203, Springer, New York, 2001, ISBN: 0-387-95067-2, xvi+238 pp.

[20] H.F. Baker, An introduction to the theory of multiply-periodic functions, Cambridge Univ. Press, Cambridge, 1907, 336 pp.

[21] J.D. Fay, Theta functions on Riemann surfaces, Lecture Notes in Math., 352, Springer-Verlag, Berlin-New York, 1973, iv+137 pp.

[22] D. Mumford, Tata lectures on theta. I: Introduction and motivation: Theta functions in one variable. Basic results on theta functions in several variables, Progr. Math., 28, Birkhäuser, Boston, MA, 1983, ISBN: 3-7643-3109-7, xiii+235 pp.; II: Jacobian theta functions and differential equations, Progr. Math., 43, Birkhäuser, Boston, 1984, ISBN: 0-8176-3110-0, xiv+272 pp.

[23] H.F. Baker, Abelian functions. Abel's theorem and the allied theory of theta functions, 1897; Reprinted in 1995: Cambridge Math. Lib., Cambridge Univ. Press, Cambridge, ISBN: 0-521-49877-5, xxxvi+684 pp.

[24] V. M. Buchstaber, V. Z. Enolskii, D. V. Leykin, "Kleinian functions, hyperelliptic Jacobians and applications", Rev. Math. Math. Phys., 10:2 (1997), 3-120.

[25] A. Nakayashiki, "On hyperelliptic Abelian functions of genus 3", J. Geom. Phys., 61:6 (2011), 961-985, arXiv: 0809.3303.

[26] K. Weierstrass, Formeln und Lehrsätze zum Gebrauche der elliptischen Functionen, Springer, Berlin, 1893.

[27] В. М. Бухштабер, Д. В. Лейкин, "Законы сложения на якобианах плоских алгебраических кривых", Тр. МИАН, 251, Наука, М., 2005, 54-126; англ. пер.: V.M. Buchstaber, D. V. Leykin, "Addition laws on Jacobians of plane algebraic curves", Proc. Steklov Inst. Math., 251 (2005), 49-120. 
[28] В. М. Бухштабер, Д. В. Лейкин, "Решение задачи дифференцирования абелевых функций по параметрам для семейств $(n, s)$-кривых", Функи. анализ и его прил., 42:4 (2008), 24-36; англ. пер.: V. M. Buchstaber, D. V. Leykin, "Solution of the problem of the differentiation of Abelian functions with respect to parameters for families of (n,s)-curves", Funct. Anal. Appl., 42:4 (2008), 268-278.

[29] J. D. Fay, Bilinear identities for theta functions, Mathematics Report № 83-168, University of Minnesota, 1983.

[30] J. Fay, "Schottky relations on $\frac{1}{2}(C-C)$ ", Theta functions - Bowdoin 1987, Part 1 (Brunswick, ME, 1987), Proc. Sympos. Pure Math., 49, Amer. Math. Soc., Providence, RI, 1989, 485-501.

[31] A. Nakayashiki, "Sigma function as a tau function", Int. Math. Res. Not. IMRN, 2010, № 3, 373-394, 22 pp.; arXiv: 0904.0846.

[32] В.М. Бухштабер, С. Ю. Шорина, "Коммутирующие дифференциальные многомерные операторы третьего порядка, задающие КдФ-иерархию”, УМН, 58:3 (2003), 187-188; англ. пер.: V. M. Bukhshtaber, S. Y. Shorina, "Commuting multidimensional third-order differential operators defining a KdV hierarchy", Russian Math. Surveys, 58:3 (2003), 610-612.

[33] В.М. Бухштабер, С. Ю. Шорина, “w-функция решения $g$-го стационарного уравнения КдФ", УМН, 58:4 (2003), 145-146; англ. пер.: V. M. Bukhshtaber, S. Y. Shorina, "The $w$-function of a solution the $g$ th stationary KdV equation", Russian Math. Surveys, 58:4 (2003), 780-781.

[34] V.M. Buchstaber, S. Yu. Shorina, "The w-function of the KdV hierarchy", Geometry, topology, and mathematical physics, Amer. Math. Soc. Transl. Ser. 2, 212, eds. V. M. Buchstaber, I. M. Krichever, Amer. Math. Soc., Providence, RI, 2004, 41-46.

[35] L. A. Dickey, Soliton equations and Hamiltonian systems, Adv. Ser. Math. Phys., 26, World Sci. Publ., River Edge, NJ, 2003, ISBN: 981-238-173-2, xii+408 pp.

[36] И. М. Кричевер, "Метод Лапласа, алгебраические кривые и нелинейные уравнения", Функи. анализ и его прил., 18:3 (1984), 43-56; англ. пер.: I. M. Krichever, "The Laplace method, algebraic curves, and nonlinear equations", Funct. Anal. Appl., 18:3 (1984), 210-223.

[37] И. М. Кричевер, С.П. Новиков, “Алгебры типа Вирасоро, римановы поверхности и структуры теории солитонов", Функи. анализ и его прил., 21:2 (1987), 46-63; англ. пер.: I. M. Krichever, S. P. Novikov, "Algebras of Virasoro type, Riemann surfaces and structures of the theory of solitons", Funct. Anal. Appl., 21:2 (1987), 126-142.

[38] P. G. Grinevich, A. Yu. Orlov, "Virasoro action on Riemann surfaces, Grassmannians, $\operatorname{det} \bar{\partial}_{J}$ and Segal-Wilson $\tau$-function", Problems of modern quantum field theory (Alushta, 1989), Res. Rep. Phys., Springer, Berlin, 1989, 86-106.

[39] H. M. Farkas, I. Kra, Riemann surfaces, Grad. Texts in Math., 71, Springer, New York-Berlin, 1980, ISBN: 0-387-90465-4, xi+337 pp.

[40] F. Klein, "Über hyperelliptische Sigmafunctionen", Math. Ann., 27:3 (1886), 431-464.

[41] F. Klein, "Über hyperelliptische Sigmafunctionen (Zweite Abhandlung)", Math. Ann., 32:3 (1888), 351-380.

[42] J. C. Eilbeck, V.Z. Enolskii, D. V. Leykin, "On the Kleinian construction of Abelian functions of canonical algebraic curves", SIDE III-symmetries and integrability of difference equations (Sabaudia, 1998), CRM Proc. Lecture Notes, 25, 2000, 121-138.

[43] В. М. Бухштабер, В. З. Энольский, Д. В. Лейкин, "Униформизация многообразий Якоби тригональных кривых и нелинейные дифференциальные уравнения", Функи. анализ и его прил., 34:3 (2000), 1-16; англ. пер.: V. M. Buchstaber, V.Z. Enolskii, D. V. Leykin, "Uniformization of Jacobi varieties of trigonal curves and nonlinear differential equations", Funct. Anal. Appl., 34:3 (2000), 159-171. 
[44] H.F. Baker, "On the hyperelliptic sigma functions", Amer. J. Math., 20:4 (1898), 301-384.

[45] J. Igusa, "Problems on Abelian functions at the time of Poincaré and some at present", Bull. Amer. Math. Soc. (N. S.), 6:2 (1982), 161-174.

[46] J. C. Eilbeck, V.Z. Enolski, J. Gibbons, "Sigma, tau and Abelian functions of algebraic curves", J. Phys. A, 43:45 (2010), 455216, 20 pp.; arXiv: 1006.5219.

[47] A. Nakayashiki, "On algebraic expression of sigma functions of $(n, s)$ curves", Asian J. Math., 14:2 (2010), 175-211; arXiv: 0803.2083.

[48] H.F. Baker, "On a system of differential equations leading to periodic functions", Acta Math., 27:1 (1903), 135-156.

[49] Ch. Athorne, "Identities for hyperelliptic $\wp$-functions of genus one, two and three in covariant form", J. Phys. A, 41:41 (2008), 415202, 20 pp.

[50] J. C. Eilbeck, V.Z. Enolski, S. Matsutani, Y. Ônishi, E. Previato, "Abelian functions for trigonal curve of genus three", Int. Math. Res. Not. IMRN, 2007, № 1, rnm 140, $38 \mathrm{pp}$.

[51] S. Matsutani, E. Previato, "Jacobi inversion on strata of the Jacobian of the $C_{r s}$ curve $y^{r}=f(x)$ ", J. Math. Soc. Japan, 60:4 (2008), 1009-1044; http://www.mittag-leffler.se/preprints/0607/files/IML-0607-41.pdf.

[52] M. England, J.C. Eilbeck, "Abelian functions associated with a cyclic tetragonal curve of genus six", J. Phys. A, 42:9 (2009), 095210, 27 pp.

[53] S. Matsutani, E. Previato, Jacobi inversion on strata of the Jacobian of the $C_{r s}$ curve $y^{r}=f(x)$, arXiv: 1006.1090.

Дж. Харнад (J. Harnad)

Université de Montréal,

Centre de recherches mathématiques;

Concordia University

E-mail: harnad@crm.umontreal.ca

В. З. Энольский (V. Z. Enolski)

Институт магнетизма НАН Украины, Киев

E-mail: V.Z.Enolskii@ma.hw.ac.uk
Поступила в редакцию

07.12 .2010 